\title{
Sample-Efficient Proper PAC Learning with Approximate Differential Privacy
}

\author{
Badih Ghazi \\ Google Research \\ Mountain View, CA, USA \\ badihghazi@gmail.com \\ Ravi Kumar \\ Google Research \\ Mountain View, CA, USA \\ ravi.k53@gmail.com
}

\author{
Noah Golowich* \\ MIT EECS \\ Cambridge, MA, USA \\ nzg@mit.edu \\ Pasin Manurangsi \\ Google Research \\ Mountain View, CA, USA \\ pasin@google.com
}

\begin{abstract}
In this paper we prove that the sample complexity of properly learning a class of Littlestone dimension $d$ with approximate differential privacy is $\tilde{O}\left(d^{6}\right)$, ignoring privacy and accuracy parameters. This result answers a question of Bun et al. (FOCS 2020) by improving upon their upper bound of $2^{O(d)}$ on the sample complexity. Prior to our work, finiteness of the sample complexity for privately learning a class of finite Littlestone dimension was only known for improper private learners, and the fact that our learner is proper answers another question of Bun et al., which was also asked by Bousquet et al. (NeurIPS 2020). Using machinery developed by Bousquet et al., we then show that the sample complexity of sanitizing a binary hypothesis class is at most polynomial in its Littlestone dimension and dual Littlestone dimension. This implies that a class is sanitizable if and only if it has finite Littlestone dimension. An important ingredient of our proofs is a new property of binary hypothesis classes that we call irreducibility, which may be of independent interest.
\end{abstract}

\section{CCS CONCEPTS}

- Theory of computation $\rightarrow$ Sample complexity and generalization bounds; Online learning theory; • Security and privacy $\rightarrow$ Formal security models.

\section{KEYWORDS}

differential privacy, PAC learning, Littlestone dimension, online learning

*Supported by a Fannie \& John Hertz Foundation Fellowship and an NSF Graduate Fellowship. This work was done while interning at Google Research

Permission to make digital or hard copies of all or part of this work for personal or classroom use is granted without fee provided that copies are not made or distributed for profit or commercial advantage and that copies bear this notice and the full citation on the first page. Copyrights for components of this work owned by others than ACM must be honored. Abstracting with credit is permitted. To copy otherwise, or republish, to post on servers or to redistribute to lists, requires prior specific permission and/or a fee. Request permissions from permissions@acm.org.

STOC '21, June 21-25, 2021, Virtual, Italy

(C) 2021 Association for Computing Machinery.

ACM ISBN 978-1-4503-8053-9/21/06.

https://doi.org/10.1145/3406325.3451028
ACM Reference Format:

Badih Ghazi, Noah Golowich, Ravi Kumar, and Pasin Manurangsi. 2021. Sample-Efficient Proper PAC Learning with Approximate Differential Privacy. In Proceedings of the 53rd Annual ACM SIGACT Symposium on Theory of Computing (STOC '21), June 21-25, 2021, Virtual, Italy. ACM, New York, NY, USA, 14 pages. https://doi.org/10.1145/3406325.3451028

\section{INTRODUCTION}

Machine learning algorithms are often trained on datasets consisting of sensitive data, such as in medical or social network applications. Protecting the privacy of the users' data is of importance, both from an ethical perspective [50] and to maintain compliance with an increasing number of laws and regulations $[25,48,49]$. The notion of differential privacy [28, 30, 54] provides a formal framework for controlling the privacy-accuracy tradeoff in numerous settings involving private data release, and it has played a central role in the development of privacy-preserving algorithms.

In the body of work on private learning algorithms, much effort has gone into developing algorithms for the private PAC model [43], namely the setting of differentially private binary classification (see Section 2.1 for a formal definition). Some papers on this topic include [5, 7, 9, 11, 19-21, 23, 33, 40, 43, 45]. A remarkable recent development $[5,21]$ in this area is the result that a hypothesis class $\mathcal{F}$ of binary classifiers is learnable with approximate differential privacy (Definition 2.2) if and only if it is online learnable, i.e., has finite Littlestone dimension (Definition 2.5). Specifically, Alon et al. [5] showed that any differentially private learning algorithm with at most constant error for a class of Littlestone dimension $d$ must use at least $\Omega\left(\log ^{\star} d\right)$ samples. Conversely, Bun et al. [21] showed that if $\mathcal{F}$ has Littlestone dimension $d$, then there is a differentially private learning algorithm for $\mathcal{F}$ with error $\alpha>0$ using $2^{O(d)} / \alpha$ samples. ${ }^{1}$

\subsection{Results}

In this paper, we resolve two open questions posed by Bun et al. [21] and Bousquet et al. [18]: first, we introduce a new private learning algorithm with sample complexity polynomial in the Littlestone dimension $d$ of the class $\mathcal{F}$, thus improving exponentially on the $2^{O(d)}$ bound from [21]. Answering a second question of [21],

\footnotetext{
${ }^{1}$ This bound ignores the dependence on the privacy parameters $\varepsilon, \delta$. Moreover, it applies to the realizable setting; a slightly weaker bound was shown in [21] for the agnostic setting.
} 
we show how to make our private learner proper (whereas the learner from [21] was improper). Whether privately properly learning classes of finite Littlestone dimension is possible was also asked by Bousquet et al. [18, Question 1]. Theorem 1.1 states our main result:

Theorem 1.1 (Private Proper PAC learning; INFormal VerSION OF THEOREM 6.3). Let $\mathcal{F}$ be a class of hypotheses $f: \mathcal{X} \rightarrow$ $\{-1,1\}$, of Littlestone dimension $d$. For any $\varepsilon, \delta, \alpha \in(0,1)$, for some $n=\tilde{O}\left(\frac{d^{6}}{\varepsilon \alpha^{2}}\right)$, there is an $(\varepsilon, \delta)$-differentially private algorithm which, given $n$ i.i.d. samples from any realizable distribution $P$ on $\mathcal{X} \times\{-1,1\}$, with high probability outputs a classifier $\hat{f} \in \mathcal{F}$ with classification error over $P$ at most $\alpha$.

The theorem statement above treats the case where the distribution $P$ over $\mathcal{X} \times\{-1,1\}$ is realizable, namely that there exists some $f^{\star} \in \mathcal{F}$ so that $P$ is supported on pairs $\left(x, f^{\star}(x)\right)$. A generic reduction of [3] allows us to show essentially the same sample complexity bound as in Theorem 1.1 for the non-realizable (i.e., agnostic) setting (see Corollary 6.4). We also remark that it is impossible to obtain a sample complexity bound better than $n=O(d)$ in the context of Theorem 1.1 if we insist that the bound depends on the class $\mathcal{F}$ only through the Littlestone dimension $d$. This follows because for any $d \in \mathbb{N}$, there are classes $\mathcal{F}$ whose Littlestone and VC dimensions are both equal to $d$ (for instance, the class of all binary hypotheses on $d$ points), and it is well-known that the VC dimension characterizes the sample complexity of learning a class (in the absence of privacy) [55].

The question posed in $[18,21]$ (and answered by Theorem 1.1) of whether classes of finite Littlestone dimension have private proper learners is motivated by a connection between proper private learning and private query release established in [18]. The problem of private query release, or sanitization $[9,17]$, for a class $\mathcal{F}$ has an extensive history, described in Section 1.2. It is defined as follows: given $\alpha>0$, a sanitizer with sample complexity $n \in \mathbb{N}$ is given as input a dataset $S=\left\{\left(x_{1}, y_{1}\right), \ldots,\left(x_{n}, y_{n}\right)\right\} \in(\mathcal{X} \times\{-1,1\})^{n}$ The sanitizer must output a function Est $: \mathcal{F} \rightarrow[0,1]$, which is differentially private for the input $S$, so that with high probability, for each $f \in \mathcal{F},\left|\operatorname{Est}(f)-\operatorname{err}_{S}(f)\right| \leqslant \alpha$, where $\operatorname{err}_{S}(f):=\frac{1}{n} \cdot \mid\{i \in$ $\left.[n]: f\left(x_{i}\right) \neq y_{i}\right\} \mid$. Bousquet et al. [18] showed that the existence of a private proper learner for a class $\mathcal{F}$ implies the existence of a sanitizer for $\mathcal{F}$; as a corollary of their result and of Theorem 1.1 we therefore obtain the following:

Corollary 1.2 (Private QUERY RELEASE; INFORMAL VERSION OF Corollary 6.6). Let $\mathcal{F}$ be a class of hypotheses $f: \mathcal{X} \rightarrow\{-1,1\}$ of Littlestone dimension $d$ and dual Littlestone dimension $d^{\star}$. For any $\varepsilon, \delta, \alpha \in[0,1]$, there is an $(\varepsilon, \delta)$-differentially private algorithm that for some $n=\operatorname{poly}\left(d, d^{\star}, 1 / \varepsilon, 1 / \alpha, \log 1 / \delta\right)$, takes as input a dataset $S$ of size $n$ and outputs a function Est $: \mathcal{F} \rightarrow[0,1]$ so that with high probability, for all $f \in \mathcal{F},\left|\operatorname{Est}(f)-\operatorname{err}_{S}(f)\right| \leqslant \alpha$.

It is known that the dual Littlestone dimension $d^{\star}$ of a class $\mathcal{F}$ is finite if and only if the Littlestone dimension $d$ is finite; in fact, we have $d^{\star} \leqslant 2^{2^{d+2}}-2$ [14, Corollary 3.6]. Thus, Corollary 1.2 implies that a class $\mathcal{F}$ is sanitizable (roughly, that it has a sanitizer with sample complexity poly $(1 / \alpha)$; see Definition 2.3 for a formal version) if it has finite Littlestone dimension. The converse, namely that any sanitizable class must have finite Littlestone dimension, follows as a consequence of a result of [23], as discussed in Section 6.2. Summarizing, we have the following:

Corollary 1.3. A hypothesis class $\mathcal{F}$ is sanitizable if and only if it has finite Littlestone dimension.

Techniques: irreducibility. The main technique that allows us to both improve the exponential bound $2^{O(d)}$ on the sample complexity from [21] to a polynomial dependence, and to make the learner proper in Theorem 1.1 is a property of hypothesis classes we introduce, called irreducibility (Section 4). Roughly speaking, a binary hypothesis class $\mathcal{G}$ of Littlestone dimension $d$ on domain $\mathcal{X}$ is irreducible if any binary tree of bounded depth labeled by elements of $\mathcal{X}$ has a leaf such that the restriction of $\mathcal{G}$ to that leaf still has Littlestone dimension $d$. The exponential sample complexity bound in [21] arises (in part) for the following reason: the main sub-procedure in their algorithm operates in a sequence of $d=\operatorname{Ldim}(\mathcal{F})$ steps, maintaining a class of candidate hypotheses; at the end of the $d$ steps, this class will have Littlestone dimension 0 (i.e., consists of a single hypothesis), and will be the hypothesis output by the sub-procedure. Each of these $d$ steps decreases the Littlestone dimension of the class of candidate hypotheses by 1 and increases the number of samples needed by a constant factor, leading to $2^{O(d)}$ samples overall. The notion of irreducibility allows us to show that certain intermediate classes of candidate hypotheses can be "sufficiently stable" to allow us to output a hypothesis associated with the intermediate class in a private way. This allows us to avoid the exponential blowup in $d$ associated with decreasing the Littlestone dimension of the candidate hypotheses all the way to 0 . We believe that the notion of irreducibility may be useful in other applications. We provide a more detailed overview of our proofs in Section 3.

\subsection{Related Work}

Sample complexity of differentially private learning. The sample complexity of PAC learning with pure differential privacy (namely, $(\varepsilon, 0)$-differential privacy) is well-understood. The seminal work of Kasiviswanathan et al. [43] showed that a finite class $\mathcal{F}$ consisting of hypotheses $f: \mathcal{X} \rightarrow\{-1,1\}$ can be learned with pure differential privacy with sample complexity $O(\log |\mathcal{F}|)$ (in this section we omit dependence on the privacy and accuracy parameters). By the Sauer-Shelah lemma, $\log (|\mathcal{F}|) \leqslant O(\operatorname{VCdim}(\mathcal{F}) \cdot \log (|X|))$; moreover, the multiplicative gap between $\operatorname{VCdim}(\mathcal{F})$, which characterizes the sample complexity of non-private learning, and $\log |\mathcal{F}|$, can be as large as $\log |\mathcal{X}|$. To obtain a more precise result, Beimel et al. [11] introduced a complexity measure for a class $\mathcal{F}$ of binary hypotheses, known as the probabilistic representation dimension of $\mathcal{F}$, which they showed to characterize the sample complexity of (improperly) learning $\mathcal{F}$ with pure differential privacy up to a constant factor (see also [7]). Feldman and Xiao [33] showed that, in turn, the probabilistic representation dimension is characterized, up to a constant factor, by the one-way public coin communication complexity of an evaluation problem associated to $\mathcal{F}$. As a corollary of this result, they established that the sample complexity of learning $\mathcal{F}$ with pure privacy is always at least $\Omega(\operatorname{Ldim}(\mathcal{F}))$, where $\operatorname{Ldim}(\mathcal{F})$ denotes the Littlestone dimension of $\mathcal{F}$. 
The current understanding of the sample complexity of learning with approximate differential privacy (namely, $(\varepsilon, \delta)$-differential privacy with $\delta$ negligible as a function of the number of users), which is our focus in this paper, is much less complete. The class of threshold functions on a domain of size $2^{d}$, which has Littlestone dimension $d$, is known to be learnable with approximate privacy with sample complexity $O\left(\left(\log ^{\star} d\right)^{1.5}\right)$ [40], showing that the sample complexity of learning a class $\mathcal{F}$ with approximate privacy can be much less than its Littlestone dimension (see also [9, 20,23], which obtained weaker bounds). As mentioned previously, the best-known lower bound for the sample complexity of (improperly) privately learning a class of Littlestone dimension $d$ is $\Omega\left(\log ^{\star} d\right)$ [5]; our Theorem 1.1 gives the best known upper bound in terms of Littlestone dimension. In a different direction, some recent papers have investigated the sample complexity of privately learning halfspaces [8, 41, 42].

Differentially private query release. The problem of private data release (also known as sanitization; see Section 2.2 for a formal definition) for a binary hypothesis class $\mathcal{F}$ dates back to Blum et al. [17], who showed that the sample complexity of private sanitization is bounded above by $O(\operatorname{VCdim}(\mathcal{F}) \cdot \log |X|) \leqslant O(\log |\mathcal{F}| \cdot \log |X|)$. This bound was later improved to $\tilde{O}(\log |\mathcal{F}| \cdot \sqrt{\log |\mathcal{X}|})$ by Hardt and Rothblum [38], ${ }^{2}$ which is known to be essentially the best possible dependence on $|\mathcal{F}|,|\mathcal{X}|$ attainable for a broad range of values of $|\mathcal{F}|,|X|[24]$. Many works have developed more finegrained bounds on the sample complexity of sanitization in terms of geometrical properties of $\mathcal{F}[15,16,32,39,46,47]$, and several have additionally studied computational considerations for this problem [29, 31, 37, 51]. However, the upper bounds on the sample complexity of sanitization obtained by all of these works scale at least polynomially with either $\log |X|$ or $\log |\mathcal{F}|$; thus, they implicitly assume that $\mathcal{X}$ or $\mathcal{F}$ (or both) is finite. In addition to being of purely theoretical interest, establishing sample complexity bounds with no explicit dependence on $|\mathcal{X}|,|\mathcal{F}|$ (and thus which can apply when $|X|$ and $|\mathcal{F}|$ are infinite) could lead to significant gains even in cases when they are finite since in many natural settings, $|X|,|\mathcal{F}|$ are exponentially large in parameters such as dimensionality of the data. The question of removing the poly $\log |\mathcal{F}|$ factors in existing bounds has also been asked in [54]: Questions 5.24 and 5.25 in [54] ask for a characterization of the sample complexity of sanitization up to "small" approximation factors. In the proof of Corollary 1.3 it is established that the sample complexity of sanitizing a class of Littlestone dimension $d$ is between $\Omega\left(\log ^{\star} d\right)$ and $2^{O\left(2^{d}\right)}$. This gap is definitely not "small" by any means, but for infinite $|\mathcal{F}|,|X|$, it is the first finite approximation factor to the best of our knowledge.

Online learning and Littlestone dimension. The Littlestone dimension of a hypothesis class $\mathcal{F}$ is known to be equal to the optimal mistake bound in the realizable setting of online learning [44, 52]. Moreover, it characterizes the optimal regret of an online learning algorithm in the agnostic setting up to a logarithmic factor: the optimal regret $\operatorname{Reg}(T)$ for an online learning algorithm with respect to a class of Littlestone dimension $d$ satisfies $\operatorname{Reg}(T)=\Theta(\sqrt{d T})[4,13,52]$. Therefore, Theorem 1.1 implies that the sample complexity of privately learning a binary hypothesis

\footnotetext{
${ }^{2}$ The $\tilde{O}$ hides factors $\operatorname{logarithmic}$ in $\log |\mathcal{F}|$ and $\log |X|$.
}

class $\mathcal{F}$ is bounded above by a polynomial in the sample complexity of online learning of $\mathcal{F}$ (in either the realizable or agnostic setting).

Many prior works have investigated the connection between online and private learnability in slightly different settings from ours. Inherent stability-type properties of private learning algorithms have been used to show that certain problems have online learning algorithms [1, 5, 36, 45]. Bun [19] shows that such a reduction is not possible in a generic sense if it is required to be computationally efficient. In the opposite direction, $[2,21]$ develop differentially private algorithms to solve problems which are online learnable.

\subsection{Organization of the Paper}

In Section 2, we review some background regarding private query release, private PAC learning, and online learning. In Section 3, we outline the proof of Theorem 1.1. In Section 4 we introduce a central notion used in our proof, namely that of irreducibility, and prove some basic properties of it. In Sections 5 and 6 we prove Theorem 1.1 and its corollaries for private query release. Concluding remarks are in Section 7.

\section{PRELIMINARIES}

We will use the script notation (e.g., $\mathcal{F}, \mathcal{X}$ ) to denote sets (e.g., sets of data points or sets of binary hypotheses). For sets $\mathcal{S}, \mathcal{T}$, we write $\mathcal{S} \subset \mathcal{T}$ to mean that $\mathcal{S}$ is a (not necessarily proper) subset of $\mathcal{T}$.

\subsection{PAC Learning}

We use standard notation and terminology regarding PAC learning (see, e.g., [53]). Let $\mathcal{X}$ be an arbitrary set and let $\{-1,1\}$ be the label set. We suppose throughout the paper that $\mathcal{X} \times\{-1,1\}$ is endowed with a $\sigma$-algebra $\Sigma$. For $x \in \mathcal{X}, y \in\{-1,1\}$, let $\delta_{(x, y)}$ denote the point measure at $(x, y)$, i.e., for $A \in \Sigma, \delta_{(x, y)}(A)$ is defined to be 1 if $(x, y) \in A$, and 0 otherwise.

A hypothesis is a function $f: \mathcal{X} \rightarrow\{-1,1\}$. We write the set of all hypotheses on $\mathcal{X}$ as $\{-1,1\}^{X}$. An example is a pair $(x, y) \in$ $\mathcal{X} \times\{-1,1\}$, and for $n \in \mathbb{N}$, a dataset $S_{n}$ is a set of $n$ examples, $S_{n}:=$ $\left\{\left(x_{1}, y_{1}\right), \ldots,\left(x_{n}, y_{n}\right)\right\}$. Given such a dataset, define the empirical measure $\hat{P}_{S_{n}}:=\frac{1}{n} \sum_{i=1}^{n} \delta_{\left(x_{i}, y_{i}\right)}$ on $\mathcal{X} \times\{-1,1\}$. For a distribution $P$ on $X \times\{-1,1\}$, let $P^{n}$ be the distribution of $S_{n} \in(X \times\{-1,1\})^{n}$ consisting of $n$ i.i.d. draws from $P$.

Definition 2.1 (Error of a hypothesis). Let $P$ be a probability distribution on $\mathcal{X} \times\{-1,1\}$. The error (or loss) of a hypothesis $f: \mathcal{X} \rightarrow\{-1,1\}$ is defined as

$$
\operatorname{err}_{P}(f):=\underset{(x, y) \sim P}{\operatorname{Pr}}[f(x) \neq y] .
$$

The empirical error of a hypothesis $f$ with respect to a dataset $S_{n}$ is defined to be $\operatorname{err}_{\hat{P}_{S_{n}}}(f)$. At times we will abbreviate $\operatorname{err}_{\hat{P}_{S_{n}}}(f)$ by writing $\operatorname{err}_{S_{n}}(f)$ instead. In this paper we will consider hypothesis classes $\mathcal{F} \subset\{-1,1\}^{X}$; to avoid having to make technical measurability assumptions on $\mathcal{F}, \mathcal{X}$, we will assume throughout that $\mathcal{F}$ and $\mathcal{X}$ are countable. (We refer the reader to [26, Chapter 5] for a discussion of such assumptions in the case that countability does not hold. We remark that it is necessary to make such measurability assumptions for standard arguments (e.g., regading uniform 
convergence) to hold even in the non-private case: without such assumptions, there are (uncountably) infinite classes of VC dimension 1, which empirical risk minimization fails to learn [12].) $b\}$.

For any $x \in \mathcal{X}, b \in\{-1,1\}$, write $\left.\mathcal{F}\right|_{(x, b)}:=\{f \in \mathcal{F}: f(x)=$

\subsection{Differential Privacy and Sanitization}

While our main focus in this paper is on PAC learning, we will additionally discuss implications of our results to differentially private data release. Therefore, in the below definition of differential privacy, we allow each user's example to belong to an arbitrary set $\mathcal{Z}$ (in PAC learning we have $\mathcal{Z}=\mathcal{X} \times\{-1,1\}$ ).

Definition 2.2 (Differential privacy, [27]). Fix sets $\mathcal{Z}, \mathcal{W}$ and $n \in$ $\mathbb{N}$, and suppose $\mathcal{W}$ is countable. ${ }^{3}$ A randomized algorithm $A$ : $\mathcal{Z}^{n} \rightarrow \mathcal{W}$ is $(\varepsilon, \delta)$-differentially private if the following holds: for any datasets $S, S^{\prime} \in \mathcal{Z}^{n}$ differing in a single example and for all subsets $\mathcal{T} \subset \mathcal{W}$,

$$
\operatorname{Pr}[A(S) \in \mathcal{T}] \leqslant e^{\varepsilon} \cdot \operatorname{Pr}\left[A\left(S^{\prime}\right) \in \mathcal{T}\right]+\delta .
$$

The sanitization (or private query release) problem was introduced in [17] and has been central in many works in differential privacy:

Definition 2.3 (Sanitization, [9, 17]). Fix $n \in \mathbb{N}$ and $\alpha, \beta, \varepsilon, \delta \in$ $(0,1)$, and suppose $\mathcal{F} \subset\{-1,1\}^{\mathcal{X}}$ is a binary hypothesis class. A randomized algorithm $A:(\mathcal{X} \times\{-1,1\})^{n} \rightarrow[0,1]^{\mathcal{F}}$ is an $(n, \alpha, \beta, \varepsilon, \delta)$-sanitizer if $A$ is $(\varepsilon, \delta)$-differentially private and for all datasets $S=\left(\left(x_{1}, y_{1}\right), \ldots,\left(x_{n}, y_{n}\right)\right) \in(\mathcal{X} \times\{-1,1\})^{n}, A(S)$ outputs a function Est : $\mathcal{F} \rightarrow[0,1]$ so that with probability at least $1-\beta$, for all $f \in \mathcal{F}$,

$$
\left|\operatorname{Est}(f)-\frac{\left|\left\{i \in[n]: f\left(x_{i}\right)=y_{i}\right\}\right|}{n}\right| \leqslant \alpha .
$$

Following [18], we say that a class $\mathcal{F}$ is sanitizable if there exists a bound $n_{\mathcal{F}}(\alpha, \beta)=\operatorname{poly}(1 / \alpha, 1 / \beta)$ so that for every $\alpha, \beta>0$, there exists an algorithm $A$ on datasets of size $n=n_{\mathcal{F}}(\alpha, \beta)$ which is an $(n, \alpha, \beta, \varepsilon, \delta)$-sanitizer for some $\varepsilon=O(1)$ and $\delta$ negligible as a function of $n$.

\subsection{VC Dimension and Uniform Convergence}

We will denote hypothesis classes, namely subsets of $\{-1,1\}^{X}$, with the letters $\mathcal{F}, \mathcal{G}, \mathcal{H}$. A class $\mathcal{F} \subset\{-1,1\}^{X}$ is said to shatter a set $\left\{x_{1}, \ldots, x_{n}\right\} \subset \mathcal{X}$ if for each choice $\left(\varepsilon_{1}, \ldots, \varepsilon_{n}\right) \in\{-1,1\}^{n}$, there is some $f \in \mathcal{F}$ so that for all $i \in[n], f\left(x_{i}\right)=\varepsilon_{i}$.

Definition 2.4 (VC dimension). The $V C$ dimension of the class $\mathcal{F}$, denoted $\operatorname{VCdim}(\mathcal{F})$, is the largest positive integer $n$ so that $\mathcal{F}$ shatters a set of size $n$.

We need the following standard fact that finite VC dimension is a sufficient condition for uniform convergence with respect to arbitrary distributions:

Theorem 2.1 (E.G., [6], Theorems $5 \& 6$ ). Suppose that $\mathcal{F}$ is countable and $\operatorname{VCdim}(\mathcal{F})=d_{\mathrm{V}} \geqslant 1$. Then there is a constant $C_{0} \geqslant 1$

\footnotetext{
${ }^{3}$ The restriction of countability may be readily removed by fixing a $\sigma$-algebra $\Sigma$ on $\mathcal{W}$ and letting $A$ be a mapping from $\mathcal{Z}^{n}$ to the space $\Delta(\mathcal{W})$ of probability measures on the measure space $(\mathcal{W}, \Sigma)$.
}

such that for any distribution $P$ on $\mathcal{X} \times\{-1,1\}$ and any $\gamma \in(0,1)$, it holds that

$$
\operatorname{Pr}_{S_{n} \sim P^{n}}\left[\sup _{f \in \mathcal{F}}\left|\operatorname{err}_{P}(f)-\operatorname{err}_{\hat{P}_{S_{n}}}(f)\right|>C_{0} \sqrt{\frac{d_{\mathrm{V}}+\log 1 / \gamma}{n}}\right] \leqslant \gamma .
$$

For a class $\mathcal{F} \subset\{-1,1\}^{\mathcal{X}}$ and a distribution $P$ on $\mathcal{X} \times\{-1,1\}$, define

$$
\mathcal{F}_{P, \alpha}:=\left\{f \in \mathcal{F}: \operatorname{err}_{P}(f) \leqslant \alpha\right\} .
$$

Note that for any $0 \leqslant \alpha \leqslant \beta \leqslant 1$, we have $\mathcal{F}_{P, \alpha} \subset \mathcal{F}_{P, \beta}$.

For any $\gamma>0$ and $n \in \mathbb{N}$, write $\alpha(n, \gamma):=C_{0} \sqrt{\frac{d_{\mathrm{V}}+\log 1 / \gamma}{n}}$, so that by Theorem 2.1 we have that

$$
\operatorname{Pr}_{S_{n}}\left[\sup _{f \in \mathcal{F}}\left|\operatorname{err}_{P}(f)-\operatorname{err}_{\hat{P}_{S_{n}}}(f)\right|>\alpha(n, \gamma)\right] \leqslant \gamma .
$$

Note that, under the event $\sup _{f \in \mathcal{F}}\left|\operatorname{err}_{P}(f)-\operatorname{err}_{\hat{P}_{S_{n}}}(f)\right| \leqslant \alpha_{0}$, we have that, for each $\alpha \in[0,1]$,

$$
\mathcal{F}_{\hat{P}_{S_{n}}, \alpha-2 \alpha_{0}} \subset \mathcal{F}_{P, \alpha-\alpha_{0}} \subset \mathcal{F}_{\hat{P}_{S_{n}}, \alpha} .
$$

Given a class $\mathcal{F} \subset\{-1,1\}^{X}$, its dual class, denoted by $\mathcal{F}^{\star}$, is defined as follows: $\mathcal{F}^{\star} \subset\{-1,1\}^{\mathcal{F}}$ and is indexed by $\mathcal{X}$. For each $x \in \mathcal{X}$, the corresponding function in $\mathcal{F}^{\star}$ is the function $x: \mathcal{F} \rightarrow\{-1,1\}$, defined by $x(f):=f(x)$. The dual VC dimension of $\mathcal{F}$, denoted by $\operatorname{VCdim}^{\star}(\mathcal{F})$, is the $\mathrm{VC}$ dimension of $\mathcal{F}^{\star}$ : i.e., $\operatorname{VCdim}^{\star}(\mathcal{F}):=\operatorname{VCdim}\left(\mathcal{F}^{\star}\right)$.

\subsection{Littlestone Dimension}

To introduce the Littlestone dimension, we need some notation regarding binary trees. For a positive integer $t$ and a sequence $b_{1}, \ldots, b_{t}, \ldots \in\{-1,1\}$, write $b_{1: t}:=\left(b_{1}, \ldots, b_{t}\right)$. As a convention, let $b_{1: 0}$ denote the empty sequence. For $n \in \mathbb{N}$, an $\mathcal{X}$-valued binary tree $\mathbf{x}$ of depth $n$ is a collection of partial functions $\mathbf{x}_{t}$ : $\{-1,1\}^{t-1} \rightarrow \mathcal{X}$ for $1 \leqslant t \leqslant n$, each with nonempty domain, so that for all $b_{1: t}$ in the domain of $\mathbf{x}_{t+1}, b_{1: t-1}$ is in the domain of $\mathbf{x}_{t}$ and $\left(b_{1}, \ldots, b_{t-1},-b_{t}\right)$ is in the domain of $\mathbf{x}_{t+1}$. If $\mathbf{x}_{t}$ is a total function for all $t$, then we say that $\mathbf{x}$ is a complete tree; otherwise, we say that $\mathbf{x}$ is incomplete. By default we will use the term "tree" to refer to a complete binary tree; when we wish to refer to incomplete trees (or the notion of generalized trees in Definition 4.4), we will use the appropriate adjective.

Associated with each sequence $b_{1: t} \in\{-1,1\}^{t}$ so that either $t=0$ or $b_{1: t-1}$ is in the domain of $\mathbf{x}_{t}$, for some $1 \leqslant t \leqslant n$, is a node of the (possibly incomplete) tree. We say that this node is a leaf if $b_{1: t}$ is not in the domain of $\mathbf{x}_{t+1}$; in particular, for complete trees, the nodes associated to each $b_{1: n} \in\{-1,1\}^{n}$ are the leaves. Suppose $b_{1: t-1}$ is in the domain of $\mathbf{x}_{t}$, for some $t$; then the node associated with $b_{1: t-1}$ is not a leaf, and we say that this node is labeled by $\mathbf{x}_{t}\left(b_{1: t-1}\right)$. For any such non-leaf node $v$, the two nodes associated with $\left(b_{1}, \ldots, b_{t-1},-1\right)$ and $\left(b_{1}, \ldots, b_{t-1}, 1\right)$ are the children of $v$ corresponding to the bits -1 and 1 , respectively. Note that a node is a leaf if and only if it has no children. Note also that any non-leaf node has exactly two children.

A class $\mathcal{F} \subset\{-1,1\}^{X}$ is said to shatter a (complete) tree $\mathbf{x}$ of depth $n$ if for all sequences $\left(b_{1}, \ldots, b_{n}\right) \in\{-1,1\}^{n}$, there is some $f \in \mathcal{F}$ so that for each $t \in[n], f\left(\mathbf{x}_{t}\left(b_{1: t-1}\right)\right)=b_{t}$. 
Definition 2.5 (Littlestone dimension). The Littlestone dimension of a class $\mathcal{F} \subset\{-1,1\}^{X}$ is the largest positive integer $n$ so that there exists a tree $\mathbf{x}$ of depth $n$ that is shattered by $\mathcal{F}$.

The Littlestone dimension is known to exactly characterize the optimal mistake bound for online learnability of the class $\mathcal{F}$ in the realizable setting [44], as well as to characterize the optimal regret bound for online learnability of $\mathcal{F}$ in the agnostic setting up to a logarithmic factor [13].

Similar to the case for VC dimension, the dual Littlestone dimension of a class $\mathcal{F}$, denoted by $\operatorname{Ldim}^{\star}(\mathcal{F})$, is the Littlestone dimension of $\mathcal{F}^{\star}$ : i.e., $\operatorname{Ldim}^{\star}(\mathcal{F}):=\operatorname{Ldim}\left(\mathcal{F}^{\star}\right)$.

\section{PROOF OVERVIEW}

In this section we overview the proof of Theorem 1.1. The proof is in two parts:

(1) The first part is a private improper learner, PolyPriLearn (Algorithm 2), with sample complexity $\tilde{O}\left(\frac{d^{6}}{\varepsilon \alpha^{2}}\right)$. The hypothesis $\hat{f} \in\{-1,1\}^{X}$ output by PolyPriLearn also satisfies an additional property, namely, it is associated with an irreducible subclass of $\mathcal{F}$ (a notion that we introduce and explain below), with high probability.

(2) The second part is a technique, PolyPriPropLearn (Algorithm 3), to convert the improper learner from the first part to a proper learner using the irreducibility property of the hypothesis $\hat{f}$.

We now elaborate further on the two parts of the proof.

Part 1: Improper learner and irreducibility. Besides allowing us to convert an improper learner to a proper one, the notion of irreducibility is central in allowing us to find a private improper learner for $\mathcal{F}$ with sample complexity polynomial in $\operatorname{Ldim}(\mathcal{F})$. Before defining irreducibility and explaining how it is useful, we first outline the overall approach. Given a dataset $S_{n}=\left\{\left(x_{1}, y_{1}\right), \ldots,\left(x_{n}, y_{n}\right)\right\}$ drawn i.i.d. from some distribution $P$ over $X \times\{-1,1\}$, we will find several subclasses $\hat{\mathcal{G}}_{1}, \ldots, \hat{\mathcal{G}}_{J} \subset \mathcal{F}$, for some $J \in \mathbb{N}$ so that for each $1 \leqslant j \leqslant J, \hat{\mathcal{G}}_{j}$ consists entirely of functions with low empirical error on the dataset $S_{n}$ (this task is performed by the sub-routine ReduceTree (Algorithm 1) of PolyPriLearn). We will then consider the SOA classifier ${ }^{5}$ for each subclass $\hat{\mathcal{G}}_{j}$; the SOA classifier for a class $\mathcal{G}$, denoted by $\mathrm{SOA}_{\mathcal{G}} \in\{-1,1\}^{\mathcal{X}}$, is defined as follows: for $x \in \mathcal{X}, \operatorname{SOA}_{\mathcal{G}}(x)=1$ if $\operatorname{Ldim}\left(\left.\mathcal{G}\right|_{(x, 1)}\right) \geqslant \operatorname{Ldim}\left(\left.\mathcal{G}\right|_{(x,-1)}\right)$, and $\operatorname{SOA}_{\mathcal{G}}(x)=-1$ otherwise. The crux of the proof rests on two facts:

(a) There are $d+1$ "special" classifiers $\sigma_{1}^{\star}, \ldots, \sigma_{d+1}^{\star} \in\{-1,1\}^{\mathcal{X}}$ (which depend on $P$ but not any particular dataset) so that with high probability, at least one of $\mathrm{SOA}_{\hat{\mathcal{G}}_{1}}, \ldots, \mathrm{SOA}_{\hat{\mathcal{G}}_{J}}$ is equal to one of $\sigma_{1}^{\star}, \ldots, \sigma_{d+1}^{\star}$.

(b) For each class $\hat{\mathcal{G}}_{j}$ that is found in the sub-routine ReduceTree, with high probability it holds that $\mathrm{SOA}_{\hat{\mathcal{G}}_{j}}$ has low population error (i.e., $\operatorname{err}_{P}\left(\mathrm{SOA}_{\hat{\mathcal{G}}_{j}}\right)$ is small).

If properties (a) and (b) are given, then the construction of a private learner is fairly straightforward: if $J$ were a constant, then we

\footnotetext{
${ }^{4}$ As a general convention we use a hat for quantities that depend on the dataset.

${ }^{5}$ As an aside, the SOA classifier achieves the optimal mistake bound in the realizable

setting of online learning $[44,52]$.
}

could draw $m=\tilde{O}(d)$ independent datasets $S_{n}^{(1)}, \ldots, S_{n}^{(m)}$ and use the private stable histogram of [22, Proposition 2.20] together with property (a) to privately output some $\mathrm{SOA}_{\mathcal{G}} \in\{-1,1\}^{X}$ that belongs to $\left\{\mathrm{SOA}_{\hat{\mathcal{G}}_{1}^{(i)}}, \ldots, \mathrm{SOA}_{\hat{\mathcal{G}}_{J}^{(i)}}\right\}$ for many of the independent datasets $S_{n}^{(i)}$ (we denote the subclasses corresponding to the $i$ th dataset, $i \in[m]$, by $\hat{\mathcal{G}}_{j}^{(i)}$, for $j \in[J]$ ). By property (b), such $\mathrm{SOA}_{\mathcal{G}}$ would then have low population error. As it turns out, we will only be able to guarantee that $J=2^{\tilde{O}\left(d^{2}\right)}$; we can still guarantee sample complexity polynomial in $d$, though, by using a variant of the stable histogram based on the exponential mechanism [35] in Algorithm 2. This will necessitate an increase in $m$ by a factor of $\log \left(2^{\tilde{O}\left(d^{2}\right)}\right)$, so that we draw a total of $m=\tilde{O}\left(d^{3}\right)$ independent datasets; each will be of size $\tilde{O}\left(d^{3}\right)$, leading to the overall sample complexity bound of $\tilde{O}\left(d^{6}\right)$.

Next we discuss the proofs of properties (a) and (b) of the subclasses $\hat{\mathcal{G}}_{1}, \ldots, \hat{\mathcal{G}}_{J}$ that the sub-routine ReduceTree outputs. The proofs of both of these properties depend on irreducibility, which we now define. We say that a hypothesis class $\mathcal{G} \subset\{-1,1\}^{X}$ is irreducible if for any $x \in \mathcal{X}$, it holds that for some $b \in\{-1,1\}$, we have $\operatorname{Ldim}\left(\left.\mathcal{F}\right|_{(x, b)}\right)=\operatorname{Ldim}(\mathcal{F})$. Definition 4.1 introduces the generalization of $k$-irreducibility for all $k \in \mathbb{N}$ (irreducibility corresponds to 1-irreducibility), but in this section we exhibit the main ideas behind the proof using $k=1$. To explain how we obtain property (a), first suppose that the following holds, for some fixed $\alpha_{\Delta}<\alpha_{0}$ :

$$
\begin{aligned}
& \text { With high probability over the sample } S_{n} \text {, it holds that } \\
& \qquad \operatorname{Ldim}\left(\mathcal{F}_{\hat{P}_{S_{n}}, \alpha}\right)=\operatorname{Ldim}\left(\mathcal{F}_{\hat{P}_{S_{n}}, \alpha-\alpha_{\Delta}}\right) \\
& \text { and } \mathcal{F}_{\hat{P}_{S_{n}}, \alpha-\alpha_{\Delta}} \text { is irreducible. }
\end{aligned}
$$

By Theorem 2.1 and (1) with $\alpha_{0}=\alpha_{\Delta} / 2$, as long as $n \geqslant \tilde{\Omega}\left(\frac{d}{\alpha_{\Delta}^{2}}\right)$, then with high probability we have $\mathcal{F}_{\hat{P}_{S_{n}}, \alpha-\alpha_{\Delta}} \subset \mathcal{F}_{P, \alpha-\alpha_{\Delta} / 2} \subset$ $\mathcal{F}_{\hat{P}_{S_{n}}, \alpha}$, and so

$$
\operatorname{Ldim}\left(\mathcal{F}_{P, \alpha-\alpha_{\Delta} / 2}\right)=\operatorname{Ldim}\left(\mathcal{F}_{\hat{P}_{S_{n}}, \alpha-\alpha_{\Delta}}\right),
$$

by (A). Using irreducibility of $\mathcal{F}_{P, \alpha-\alpha_{\Delta}}$ and (2), it is straightforward to show (Lemma 4.2) that

$$
\operatorname{SOA}_{\mathcal{F}_{P, \alpha-\alpha_{\Delta} / 2}}=\operatorname{SOA}_{\mathcal{F}_{\hat{P}_{S_{n}}, \alpha-\alpha_{\Delta}}} .
$$

Thus, we have shown, assuming (A), that a quantity that can be computed from the empirical data, namely $\mathrm{SOA}_{\mathcal{F}_{\hat{P}_{S_{n}}, \alpha-\alpha_{\Lambda}}}$, is equal with high probability to a fixed quantity, namely $\mathrm{SOA}_{\mathcal{F}_{P, \alpha-\alpha_{\Lambda} / 2}}$, which we may take to be, say $\sigma_{1}^{\star}$, in property (a).

Of course, we must also deal with the case where (A) does not hold. There are two possible reasons for this: the first is that $\operatorname{Ldim}\left(\mathcal{F}_{\hat{P}_{S_{n}}, \alpha-\alpha_{\Delta}}\right)<\operatorname{Ldim}\left(\mathcal{F}_{\hat{P}_{S_{n}}, \alpha}\right)$. In this case, as long as $\alpha_{\Delta}$ is sufficiently small, we may replace $\alpha$ with $\alpha-\alpha_{\Delta}$ and recurse (i.e., check if (A) holds with the new value of $\alpha$, and act accordingly). Since $\operatorname{Ldim}\left(\mathcal{F}_{\hat{P}_{S_{n}}, \alpha}\right) \leqslant \operatorname{Ldim}(\mathcal{F}) \leqslant d$, the Littlestone dimension can decrease at most $d$ times and therefore it is sufficient to choose $\alpha_{\Delta} \approx \alpha / d$ (and so we may take $n=\tilde{O}\left(d^{3}\right)$ ).

The other reason that (A) may fail to hold is that $\mathcal{F}_{\hat{P}_{S_{n}}, \alpha-\alpha_{\Delta}}$ is not irreducible. In such a case, by definition of irreducibility, there 
exists some $x \in \mathcal{X}$ so that $\max \left\{\operatorname{Ldim}\left(\mathcal{F}_{\hat{P}_{S_{n}}, \alpha-\alpha_{\Delta}} \mid(x, 1)\right), \operatorname{Ldim}\left(\left.\mathcal{F}_{\hat{P}_{S_{n}}, \alpha-\alpha_{\Delta}}\right|_{(x,-1)}\right)\right\}<\operatorname{Ldim}(\mathcal{F})$. The idea is to now make $t w o$ recursive calls, replacing $\alpha$ with $\alpha-\alpha_{\Delta}$ (as before) and using each of the classes $\left.\mathcal{F}\right|_{(x, 1)}$ and $\left.\mathcal{F}\right|_{(x,-1)}$ in place of $\mathcal{F}$. A clear issue with this approach is that $x$ may depend on the dataset $S_{n}$, and so the crucial "stability" property of (3) may fail to hold in the recursive call, even if (A) holds with the new $\alpha$ and for the class $\left.\mathcal{F}\right|_{(x, \pm 1)}$. It turns out that we can amend this issue by replacing irreducibility in (A) with the stronger property of $k$-irreducibility for $k>1$; the details can be found in Sections 4 and 5.1.

This process of decreasing the Littlestone dimension by at least 1 and then making some number of "recursive" calls results in a tree with at most $2^{\tilde{O}\left(d^{2}\right)}$ leaves (Definition 4.4 describes the specific tree structure). Each of these leaves determines a class $\hat{\mathcal{G}}_{j}$, and using a generalization of (3), we can ensure that the classes $\hat{\mathcal{G}}_{j}$ satisfy property (a). Moreover, we will be able to ensure that for a sufficiently large integer $k$, each $\hat{\mathcal{G}}_{j}$ is $k$-irreducible; this will be enough to show that property (b) above holds via a fairly straightforward argument (carried out in Lemma 4.3 and Claim 5.9).

Part 2: Making the improper learner proper. Let $\mathrm{SOA}_{\hat{\mathcal{G}}} \in\{-1,1\}^{X}$ be the classifier output by the private improper learner PolyPriLearn described above. The idea to make this learner proper is to find a small set $\hat{\mathcal{H}} \subset \mathcal{F}$ (in particular, of size bounded by $O\left(\operatorname{VCdim}^{\star}(\mathcal{F}) / \alpha^{2}\right)$ ), such that for any distribution $Q$ over $\mathcal{X}$, there is some $\hat{h} \in \hat{\mathcal{H}}$ such that $\operatorname{Pr}_{x \sim Q}\left[\operatorname{SOA}_{\hat{\mathcal{G}}}(x) \neq \hat{h}(x)\right] \leqslant \alpha$. In particular, this holds for $Q=P$, the true population distribution. Thus, since the improper learner from above guarantees that $\mathrm{SOA}_{\hat{\mathcal{G}}}$ has low population error under $P$ with high probability, we can choose some $\hat{h} \in \hat{\mathcal{H}}$ with not much higher error using the exponential mechanism on a fresh set of samples of size roughly $\log |\hat{\mathcal{H}}| \leqslant \tilde{O}\left(\log \operatorname{VCdim}^{\star}(\mathcal{F})\right) \leqslant$ $\tilde{O}(\operatorname{VCdim}(\mathcal{F}))$ (this is explained in detail in PolyPriPropLearn, Algorithm 3).

It remains to show the existence of a small $\hat{\mathcal{H}} \subset \mathcal{F}$. To do so, we consider the zero-sum game with action spaces $\mathcal{F}$ and $\mathcal{X}$, where the row player chooses $h \in \mathcal{F}$, the column player chooses $x \in \mathcal{X}$, and the value of the game is $\mathbf{1}\left[h(x) \neq \mathrm{SOA}_{\hat{\mathcal{G}}}(x)\right]$. By von Neumann's minimax theorem, we have

$$
\begin{aligned}
& \inf _{D \in \Delta(\mathcal{F})} \sup _{P \in \Delta(X)} \mathbb{E}_{X \sim P, h \sim D}\left[1\left[\operatorname{SOA}_{\hat{\mathcal{G}}}(x) \neq h(x)\right]\right] \\
& =\sup _{P \in \Delta(\mathcal{X})} \inf _{D \in \Delta(\mathcal{F})} \mathbb{E}_{X \sim P, h \sim D}\left[1\left[\operatorname{SOA}_{\hat{\mathcal{G}}}(x) \neq h(x)\right]\right] .
\end{aligned}
$$

Using the fact that the class $\hat{\mathcal{G}}$ corresponding to the classifier $\mathrm{SOA}_{\hat{\mathcal{G}}}$ output by PolyPriLearn is $k$-irreducible for a sufficiently large integer $k$, we show in [34, Lemma 6.1] that the right-hand side of (4) is bounded above by the desired accuracy $\alpha$. Thus the same holds for the left-hand side of (4). Now take a distribution $\hat{D} \in \Delta(\mathcal{F})$ attaining the infimum on the left-hand side of (4); using a uniform convergence argument applied to the dual class of $\mathcal{F}$ (Lemma 6.1), we may choose a multiset $\hat{\mathcal{H}} \subset \mathcal{F}$ of size $O\left(\operatorname{VCdim}^{\star}(\mathcal{F}) / \alpha^{2}\right)$ so that the uniform distribution over $\hat{\mathcal{H}}$ comes close to the infimum on the left-hand side of (4). Such an $\hat{\mathcal{H}}$ satisfies the property we desired.

\section{IRREDUCIBILITY}

In this section we make a definition which is central to our algorithm and its analysis, namely that of irreducibility of a hypothesis class. We then state some lemmas establishing basic properties of irreducible classes; proofs for this section may be found in the full version of this paper [34].

Fix some set $\mathcal{X}$ and a space $\mathcal{G}$ of hypotheses on $\mathcal{X}$. For any $x \in \mathcal{X}, b \in\{-1,1\}$, set

$$
\left.\mathcal{G}\right|_{(x, b)}:=\{f \in \mathcal{G}: f(x)=b\} .
$$

For a set $S=\left\{\left(x_{1}, b_{1}\right), \ldots,\left(x_{n}, b_{n}\right)\right\}$, similarly set

$$
\left.\mathcal{G}\right|_{S}:=\left.\bigcap_{i \in[n]} \mathcal{G}\right|_{\left(x_{i}, b_{i}\right)}=\left\{f \in \mathcal{G}: f\left(x_{i}\right)=b_{i} \forall i \in[n]\right\} .
$$

For $S$ as above, we will at times abuse notation slightly and write $\left.\mathcal{G}\right|_{S}=\left.\mathcal{G}\right|_{\left(x_{1}, b_{1}\right), \ldots,\left(x_{n}, b_{n}\right)}$.

Definition 4.1 (Irreducibility). A class $\mathcal{G}$ is $k$-irreducible if for any depth- $k$ tree $\mathbf{x}$, there is some choice of bits $b_{1}, \ldots, b_{k} \in\{-1,1\}$ such that

$$
\operatorname{Ldim}\left(\left.\mathcal{G}\right|_{\left(\mathbf{x}_{1}, b_{1}\right),\left(\mathbf{x}_{2}\left(b_{1}\right), b_{2}\right) \ldots,\left(\mathbf{x}_{k}\left(b_{1: k-1}\right), b_{k}\right)}\right)=\operatorname{Ldim}(\mathcal{G}) .
$$

We say that the class $\mathcal{G}$ is irreducible if it is 1-irreducible.

Note that $k$-irreducibility implies $k^{\prime}$-irreducibility for $k^{\prime}<k$.

The following lemma shows that $k$-irreducibility satisfies a sort of "monotonicity" property among classes of the same Littlestone dimension.

Lemma 4.1. Suppose $\mathcal{H} \subset \mathcal{G}$, and $\operatorname{Ldim}(\mathcal{H})=\operatorname{Ldim}(\mathcal{G})$. If $\mathcal{H}$ is $k$-irreducible, then so is $\mathcal{G}$.

We next define the SOA classifier associated with a function class $\mathcal{G}$; the choice of name is due to its similarity to the classifiers used in the standard optimal algorithm (SOA) in online learning [13, 44].

Definition 4.2 (SOA classifier). For a class $\mathcal{G}$, define the function $\mathrm{SOA}_{\mathcal{G}}: \mathcal{X} \rightarrow\{-1,1\}$ as follows:

$$
\operatorname{SOA}_{\mathcal{G}}(x):=\left\{\begin{array}{l}
1 \quad: \operatorname{Ldim}\left(\left.\mathcal{G}\right|_{(x, 1)}\right) \geqslant \operatorname{Ldim}\left(\left.\mathcal{G}\right|_{(x,-1)}\right) \\
-1 \quad: \operatorname{Ldim}\left(\left.\mathcal{G}\right|_{(x, 1)}\right)<\operatorname{Ldim}\left(\left.\mathcal{G}\right|_{(x,-1)}\right) .
\end{array}\right.
$$

Lemma 4.2 establishes an important "stability-type" property satisfied by SOA classifiers of irreducible classes.

Lemma 4.2. Suppose $\mathcal{H} \subset \mathcal{G}, \operatorname{Ldim}(\mathcal{H})=\operatorname{Ldim}(\mathcal{G})$, and that $\mathcal{H}$ is irreducible. Then for all $x \in \mathcal{X}, \operatorname{SOA}_{\mathcal{H}}(x)=\operatorname{SOA}_{\mathcal{G}}(x)$.

The below lemma implies generalization bounds for the family of hypotheses $\mathrm{SOA}_{\mathcal{G}}$, for $\mathcal{G} \subset \mathcal{F}$ that are irreducible of sufficiently high order.

Lemma 4.3. For a class $\mathcal{F}$ with $\operatorname{Ldim}(\mathcal{F})=d$, set $\tilde{\mathcal{F}}_{d+1}:=\left\{\operatorname{SOA}_{\mathcal{G}}: \mathcal{G} \subset \mathcal{F}, \mathcal{G}\right.$ is nonempty $\&(d+1)$-irreducible. $\}$

Then $\operatorname{Ldim}\left(\tilde{\mathcal{F}}_{d+1}\right)=d$ as well.

Note that $\mathcal{F} \subset \tilde{\mathcal{F}}_{d+1}$, since for any $f \in \mathcal{F},\{f\}$ is $k$-irreducible for all $k \in \mathbb{N}$, and $\operatorname{SOA}_{\{f\}}=f$.

In Definitions 4.3 and 4.4 below, we generalize the notion of tree to include those in which each node may have more than two 
children. The scheme by which we label nodes is somewhat nonstandard so as to more closely correspond to the types of trees constructed in Algorithm 1 in the following section.

Definition 4.3 (Reducing arrays). A reducing array of depth $k$ is a collection of $k+1$ tuples $b^{(j)}:=\left(b_{1}^{(j)}, \ldots, b_{j \wedge k}^{(j)}\right) \in\{-1,1\}^{j \wedge k}$ for $1 \leqslant j \leqslant k+1$, which satisfy the following property: $b_{j^{\prime}}^{(j+1)}=b_{j^{\prime}}^{(j)}$ for all $j^{\prime}<j \leqslant k$, and $b_{j}^{(j+1)}=-b_{j}^{(j)}$ for $j \leqslant k^{6}{ }^{6}$

Definition 4.4 (Generalized trees). A generalized tree $\mathrm{x}$ with values in $\mathcal{X}$ of depth $d$ and branching factor $k \in \mathbb{N}$ is a rooted tree of depth at most $d$ in which each node has at most $k+1$ children. ${ }^{7}$ Nodes of the tree without children are called its leaves. Moreover, the nodes and edges of the tree are labeled as follows:

(1) Each non-leaf node $v$ is labeled with an ordered tuple of some number $k_{v} \leqslant k$ of points in $\mathcal{X}$, denoted by $\left(\mathbf{x}(v)_{1}, \ldots, \mathbf{x}(v)_{k_{v}}\right) \in$ $x^{k_{v}}$.

(2) The non-leaf node $v$ has $k_{v}+1$ children; the edge between $v$ and the $j$ th child, $1 \leqslant j \leqslant k_{v}+1$, is labeled by a tuple $b^{(j)}$, where the tuples $b^{(j)} \in\{-1,1\}^{j \wedge k_{v}}$ form a reducing array of depth $k_{v}$ (Definition 4.3).

Moreover, for any node $v$ (perhaps a leaf), define $\mathbf{a}(v) \in(X \times$ $\{-1,1\})^{\star}\left(\right.$ called the ancestor set of $v$ ) as follows: let $v^{(1)}, \ldots, v^{(t-1)}$ be the root-to-leaf path for the node $v$ and $v^{(t)}:=v$. For each $1 \leqslant i \leqslant t-1$, let $b^{(i)} \in\{-1,1\}^{k^{(i)}}$ be the label of the edge between $v^{(i)}$ and $v^{(i+1)}$, where $k^{(i)} \leqslant k_{v^{(i)}}$ is some positive integer. Then

$$
\begin{aligned}
\mathbf{a}(v):= & \left\{\left(\mathbf{x}\left(v^{(1)}\right)_{1}, b_{1}^{(1)}\right), \ldots,\left(\mathbf{x}\left(v^{(1)}\right)_{k^{(1)}}, b_{k^{(1)}}^{(1)}\right)\right\} \cup \cdots \\
& \cup\left\{\left(\mathbf{x}\left(v^{(t-1)}\right)_{1}, b_{1}^{(t-1)}\right), \ldots,\left(\mathbf{x}\left(v^{(t-1)}\right)_{k^{(t-1)}}, b_{k^{(t-1)}}^{(t-1)}\right)\right\} .
\end{aligned}
$$

The height of the node $v$ is defined to be $k^{(1)}+\cdots+k^{(t-1)}$, where $k^{(1)}, \ldots, k^{(t-1)}$ are defined given $v$ as above. Note that the height of $v$ is at least the size of (i.e., number of tuples in) the ancestor set $\mathbf{a}(v)$; the height may be even greater if there are duplicates in $\mathbf{a}(v)$. The height of the tree $\mathbf{x}$, denoted by $h t(\mathbf{x})$, is the maximum height of any node $v$ of $\mathbf{x}$. Note that we must have ht $(\mathbf{x}) \geqslant d$ if the depth of $\mathbf{x}$ is $d$. To avoid ambiguity, when we wish to refer to a generalized tree, we will always use the adjective "generalized"; "tree" will continue to mean "complete binary tree".

Lemma 4.4 below explains the choice of name "reducing array": it shows that if a class $\mathcal{G}$ is not $k$-irreducible, then there is a reducing array that can be used to "reduce the Littlestone dimension of $\mathcal{G}$ " in a certain sense. As a matter of convention, if the class $\mathcal{G}$ is empty we write $\operatorname{Ldim}(\mathcal{G})=-1$. Note also that $\operatorname{Ldim}(\mathcal{G})=0$ if and only if $\mathcal{G}$ contains a single hypothesis.

LEMMA 4.4. Suppose that $\mathcal{G}$ is not $k$-irreducible but is $(k-1)$ irreducible. Then for some $1 \leqslant k^{\prime} \leqslant k$ there is a reducing array of depth $k^{\prime}$, denoted by $b^{(1)}, \ldots, b^{\left(k^{\prime}+1\right)}$, and a sequence $x_{1}, \ldots, x_{k^{\prime}} \in$

\footnotetext{
${ }^{6}$ For real numbers $a, b$, we use the notation $a \wedge b$ and $a \vee b$ to denote $\min \{a, b\}$ and $\max \{a, b\}$ respectively.

${ }^{7}$ By depth at most $d$, we mean that the number of edges in the path from the root to any leaf is at most $d$; this aligns with the meaning of depth for binary trees in Section 2.4 .
}

$\mathcal{X}$ so that for all $j \in\left[k^{\prime}+1\right]$, we have

$$
0 \leqslant \operatorname{Ldim}\left(\left.\mathcal{G}\right|_{\left(x_{1}, b_{1}^{(j)}\right), \ldots,\left(x_{j \wedge k^{\prime}}, b_{j \wedge k^{\prime}}^{(j)}\right)}\right)<\operatorname{Ldim}(\mathcal{G}) .
$$

Lemma 4.5 is a key part of the proof that the ReduceTree algorithm presented in Section 5.1 can be used together with the sparse selection protocol of Section 5.2 to generate an (improper) private learner. Roughly speaking, it gives sufficient conditions for a generalized tree $\mathbf{x}$ (which will depend on the input dataset) to have some leaf $\hat{v}$ so that for any hypothesis class $\mathcal{J}$ in a certain family of hypothesis classes, it holds that $\mathrm{SOA}_{\left.\mathcal{J}\right|_{\mathbf{a}(\hat{v})}}=\operatorname{SOA}_{\left.\mathcal{J}\right|_{S^{\star}}}$, where $S^{\star} \in(\mathcal{X} \times\{-1,1\})^{\star}$ is a collection of $(x, y)$ pairs which will not depend on the input dataset. The statement of Lemma 4.5 is in fact slightly more general (so that the preceding statement corresponds to the case $\mathcal{J}=\mathcal{J}^{\prime}$ in Lemma 4.5).

Lemma 4.5. Fix some $k, k^{\prime} \in \mathbb{N}$ with $k>k^{\prime}$ and hypothesis classes $\mathcal{H} \subset \mathcal{G} \subset\{-1,1\}^{X}$. Suppose we are given $S^{\star} \in(\mathcal{X} \times\{-1,1\})^{k-k^{\prime}}$ so that $\left.\mathcal{H}\right|_{S^{\star}}$ is $k$-irreducible, and that

$$
\operatorname{Ldim}\left(\left.\mathcal{G}\right|_{S^{\star}}\right)=\operatorname{Ldim}\left(\left.\mathcal{H}\right|_{S^{\star}}\right)=: \ell^{\star} \geqslant 0 .
$$

Suppose that $\mathbf{x}$ is a generalized tree so that $\mathrm{ht}(\mathbf{x}) \leqslant k-k^{\prime}$ and for all leaves $v$ of $\mathbf{x}, \operatorname{Ldim}\left(\left.\mathcal{G}\right|_{\mathbf{a}(v)}\right) \leqslant \ell^{\star}$. Then there is some leaf $\hat{v}$ of $\mathbf{x}$ so that $\mathrm{SOA}_{\left.\mathcal{J}\right|_{S^{\star}}}=\mathrm{SOA}_{\left.\mathcal{J}^{\prime}\right|_{\mathrm{a}(\hat{v})}}$ for all hypothesis classes $\mathcal{J}^{\prime}, \mathcal{J}$ satisfying $\mathcal{H} \subset \mathcal{J}^{\prime} \subset \mathcal{G}$ and $\mathcal{H} \subset \mathcal{J} \subset \mathcal{G}$.

Moreover, the leaf $\hat{v}$ satisfies:

(1) $\operatorname{Ldim}\left(\left.\mathcal{G}\right|_{\mathbf{a}(\hat{v})}\right)=\operatorname{Ldim}\left(\left.\mathcal{H}\right|_{\mathbf{a}(\hat{v})}\right)=\ell^{\star}$.

(2) $\left.\mathcal{H}\right|_{\mathbf{a}(\hat{v})}$ is $k^{\prime}$-irreducible.

\section{IMPROPER PRIVATE LEARNER FOR LITTLESTONE CLASSES}

In this section we establish a variant of Theorem 1.1 where the learner is only guaranteed to be improper (namely, part 1 of the proof as described in Section 3). In Section 5.1 we introduce the algorithm ReduceTree (defined in Algorithm 1), which, given a dataset $S_{n}=\left(\left(x_{1}, y_{1}\right), \ldots,\left(x_{n}, y_{n}\right)\right)$ outputs a set $\hat{\mathcal{S}}$ of hypotheses of the form $\mathrm{SOA}_{\mathcal{G}}$ for various classes $\mathcal{G} \subset \mathcal{F}$. (To aid our notation in the proofs we also have ReduceTree output a generalized tree $\hat{\mathbf{x}}$ and a set $\hat{\mathcal{L}}^{\prime}$ of leaves of $\hat{\mathbf{x}}$.) In Section 5.2 we state guarantees for a private sparse selection protocol from [35]. In Section 5.3 we will show how to use certain "stability-type" properties of the set $\hat{\mathcal{S}}$ together with the private sparse selection procedure from Section 5.2 to privately output a hypothesis which has low population error, which will establish the desired improper learner. (We will then make it proper in Section 6, thus establishing Theorem 1.1 in its entirety.)

\subsection{Building Block: ReduceTree Algorithm}

Throughout this section we fix a function class $\mathcal{F}$ and write $d:=$ $\operatorname{Ldim}(\mathcal{F})$; we also assume that a given distribution $P$ over $\mathcal{X} \times$ $\{-1,1\}$ is realizable for $\mathcal{F}$. The algorithm ReduceTree takes some $\gamma \in[0,1], n, k^{\prime} \in \mathbb{N}$ as parameters (to be specified below). It also takes as input some dataset $S_{n} \in(X \times\{-1,1\})^{n}$, which is accessed via the empirical distribution $\hat{P}_{S_{n}}$. Recall the function $\alpha(n, \gamma) \in$ $[0,1]$ defined after Theorem 2.1. Let $\alpha_{\Delta}:=6 \cdot \alpha(n, \gamma)$, and define 
$E_{\text {good }}$ to be the event

$$
E_{\text {good }}:=\left\{\sup _{f \in \mathcal{F}}\left|\operatorname{err} P(f)-\operatorname{err}_{\hat{P}_{S_{n}}}(f)\right| \leqslant \frac{\alpha_{\Delta}}{6}\right\} .
$$

Assuming that the dataset $S_{n}=\left\{\left(x_{1}, y_{1}\right), \ldots,\left(x_{n}, y_{n}\right)\right\}$ is distributed i.i.d. according to $P$, by Theorem 2.1, $\operatorname{Pr}_{S_{n} \sim P^{n}}\left[E_{\text {good }}\right] \geqslant$ $1-\gamma$.

The algorithm ReduceTree operates as follows. It starts with the class $\mathcal{F}_{\hat{P}_{S_{n}}, \alpha_{0}}$ of hypotheses with empirical error at most $\alpha_{0}$ ( $\alpha_{0}$ will be chosen so that it is less than the desired error for the output of the private learner). If it is the case that $\operatorname{Ldim}\left(\mathcal{F}_{\hat{P}_{S_{n}}, \alpha_{0}}\right)=$ $\operatorname{Ldim}\left(\mathcal{F}_{\hat{P}_{S_{n}}, \alpha_{0}-\alpha_{\Delta}}\right)$ and $\mathcal{F}_{\hat{P}_{S_{n}}, \alpha_{0}-\alpha_{\Delta}}$ is irreducible for some appropriately chosen $\alpha_{\Delta}>0$, then by Lemmas 4.1 and 4.2 , under the event $E_{\text {good }}$, the classifier $\mathrm{SOA}_{\mathcal{F}_{\hat{P}_{S_{n}}, \alpha_{0}-2 \alpha_{\Delta} / 3}}$ is "stable" in the sense that it does not "depend much" on the dataset $S_{n}$. (We leave a formalization of this statement to the proof below.) In this case we can output the set consisting of the single hypothesis, $\hat{\mathcal{S}}:=\left\{\mathrm{SOA}_{\mathcal{F}_{\hat{P}_{S_{n}}, \alpha_{0}-2 \alpha_{\Delta} / 3}}\right\}$.

If we did not terminate in the above paragraph, then one of the following two statements must hold: (1) it holds that $\operatorname{Ldim}\left(\mathcal{F}_{\hat{P}_{S_{n}}, \alpha_{0}-\alpha_{\Delta}}\right)$ $<\operatorname{Ldim}\left(\mathcal{F}_{\hat{P}_{S_{n}}, \alpha_{0}}\right)$, or (2) $\mathcal{F}_{\hat{P}_{S_{n}}, \alpha_{0}-\alpha_{\Delta}}$ is not irreducible and $\operatorname{Ldim}\left(\mathcal{F}_{\hat{P}_{S_{n}}, \alpha_{0}-\alpha_{\Delta}}\right)=\operatorname{Ldim}\left(\mathcal{F}_{\hat{P}_{S_{n}}, \alpha_{0}}\right)$. If (1) holds, then we may simply recurse, i.e., repeat the above process with $\alpha_{1}:=\alpha_{0}-\alpha_{\Delta}$ replacing $\alpha_{0}$. Otherwise, (2) holds, so (by Lemma 4.4 with $k=1$ ) we can choose some $x \in \mathcal{X}$ so that $\operatorname{Ldim}\left(\mathcal{F}_{\hat{P}_{S_{n}}, \alpha_{0}-\alpha_{\Delta}} \mid(x, b)\right)<$ $\operatorname{Ldim}\left(\mathcal{F}_{\hat{P}_{S_{n}}, \alpha_{0}-\alpha_{\Delta}}\right)=\operatorname{Ldim}\left(\mathcal{F}_{\hat{P}_{S_{n}}, \alpha_{0}}\right)$ for each $b \in\{-1,1\}$. In this case, we can repeat the above process twice (once for each $b \in\{-1,1\})$, with $\alpha_{1}:=\alpha_{0}-\alpha_{\Delta}$ replacing $\alpha_{0}$ and with $\left.\mathcal{F}\right|_{(x, b)}$ replacing $\mathcal{F}$ for each $b \in\{-1,1\}$. The point $x \in \mathcal{X}$ becomes the label of the root node of a generalized tree, with two children (which are leaves), corresponding to the bits \pm 1 . Each step $t \geqslant 1$ of the above-described recursion builds upon this generalized tree maintained by the algorithm by adding children to some of its current leaves. For technical reasons, at depth $t$ of this recursion, we will need to replace the requirement of "irreducibility" with that of " $k{ }^{\prime} \cdot 2^{t}$-irreducibility", for some $k^{\prime}$ which does not depend on $t$. The algorithm is guaranteed to terminate because with each increase in $t$, the Littlestone dimension of the current class under consideration decreases, and it can only do so at $\operatorname{most} \operatorname{Ldim}(\mathcal{F})$ times. Further details may be found in Algorithm 1 .

We say that the dataset $S_{n}$ is realizable if there is some $f \in \mathcal{F}$ so that $\operatorname{err}_{\hat{P}_{S_{n}}}(f)=0$ (this is the case with probability 1 if $S_{n} \sim P^{n}$ and $P$ is realizable). Lemma 5.1 states a basic property of the output set $\hat{\mathcal{L}}^{\prime}$ of ReduceTree.

LEMMA 5.1. Suppose the input dataset $S_{n}$ of ReduceTree is realizable. The set $\hat{\mathcal{L}}^{\prime}$ output by ReduceTree satisfies the following property: letting $t=t_{\text {final }}+1 \in[d+1]$, there is some leaf $v \in \hat{\mathcal{L}}^{\prime}$ so that $\operatorname{Ldim}\left(\hat{\mathcal{G}}\left(\alpha_{t}-\alpha_{\Delta}, v\right)\right)=\operatorname{Ldim}\left(\hat{\mathcal{G}}\left(\alpha_{t}, v\right)\right) \geqslant 0$ and $\hat{\mathcal{G}}\left(\alpha_{t}-\alpha_{\Delta}, v\right)$ is $k_{t}$-irreducible.

The proof of Lemma 5.1 may be found in the full version of this paper [34].

In order to apply Lemma 4.5 in the proof of Lemma 5.4 below, we will need an upper bound on $\mathrm{ht}(\hat{\mathbf{x}})$ for the tree $\hat{\mathbf{x}}$ output by

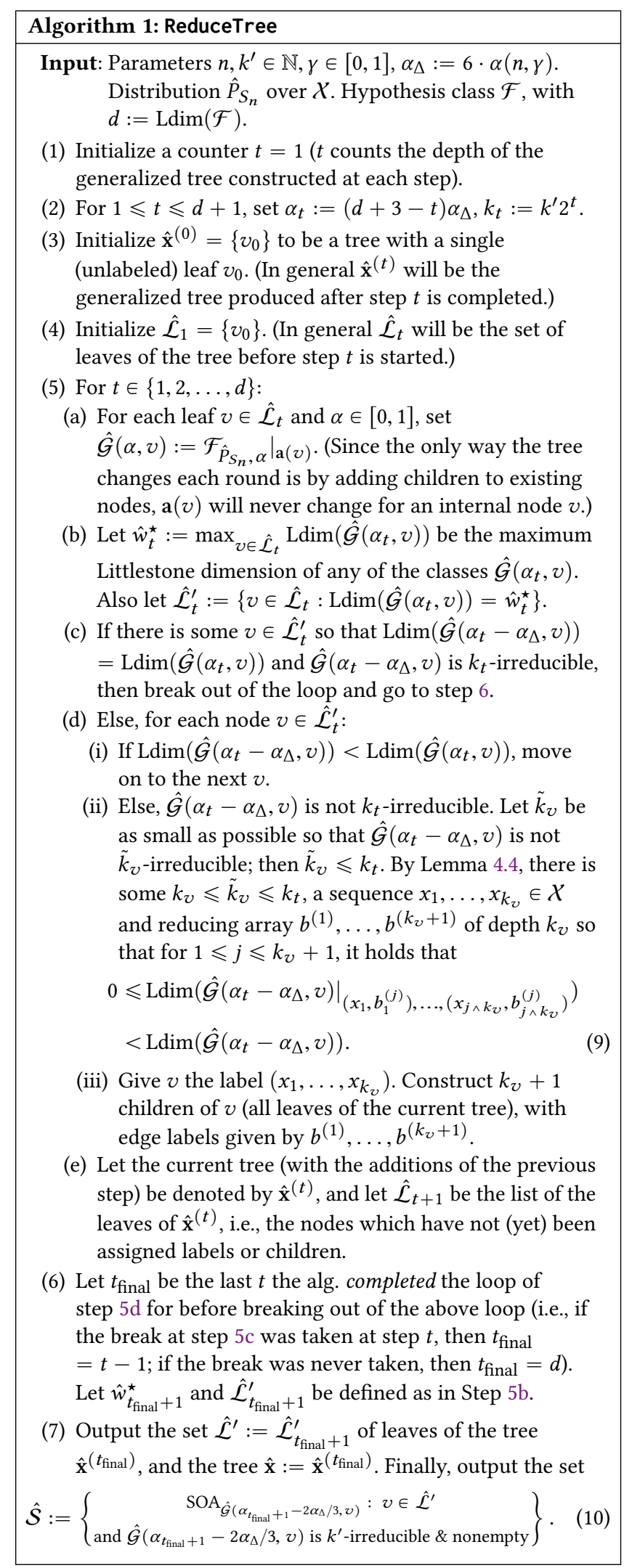


ReduceTree. Lemma 5.2 provides this upper bound; roughly speaking, the growth is exponential in $t$ (recall $k_{t}=k^{\prime} \cdot 2^{t}$ from step 2 of Algorithm 1) because the tree may grow in height by $k_{t}$ with each increase of $t$ by 1 (due to step 5(d)ii of the algorithm), and in order to satisfy the preconditions of Lemma 4.5 we need to ensure that $k_{t+1}$ is an upper bound on $\mathrm{ht}\left(\hat{\mathbf{x}}^{(t)}\right)$ for each $t$.

LEMma 5.2. For all the tree $\hat{\mathbf{x}}^{(t)}$ of Algorithm 1 satisfies ht $\left(\hat{\mathbf{x}}^{(t)}\right) \leqslant$ $k_{t+1}-k^{\prime}$. In particular, the tree $\hat{\mathbf{x}}$ satisfies ht $(\hat{\mathbf{x}}) \leqslant k_{t_{\text {final }}+1}-k^{\prime}$.

Proof. We prove that $h \mathrm{t}\left(\hat{\mathbf{x}}^{(t)}\right) \leqslant k_{t+1}-k^{\prime}=k^{\prime} \cdot 2^{t+1}-k^{\prime}$ by induction. For the base case, note that ht $\left(\hat{\mathbf{x}}^{(0)}\right)=0<2 k^{\prime}-k^{\prime}=$ $k^{\prime} \cdot 2^{1}-k^{\prime}$. Since at step 5(d)ii of the algorithm, in the $t$ th iteration, each leaf is labeled with a tuple of length at most $k_{t}$, it follows that

$$
\operatorname{ht}\left(\hat{\mathbf{x}}^{(t)}\right) \leqslant \operatorname{ht}\left(\hat{\mathbf{x}}^{(t-1)}\right)+k_{t} \leqslant k_{t}-k^{\prime}+k_{t}=k_{t+1}-k^{\prime},
$$

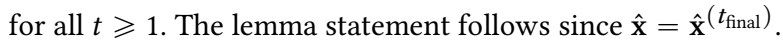

For each $\alpha \in[0,1]$ and $t \in[d+1]$, define the set:

$$
\begin{aligned}
& \mathcal{M}_{\alpha, t}:=\left\{S \in(X \times\{-1,1\})^{k_{t}-k^{\prime}}:\right. \\
& \mathcal{F}_{P, \alpha-\alpha_{\Delta} / 3} \mid s \text { is } k_{t} \text {-irreducible and nonempty, } \\
& \text { and } \left.\operatorname{Ldim}\left(\mathcal{F}_{P, \alpha-\alpha_{\Delta} / 3} \mid S\right)=\operatorname{Ldim}\left(\mathcal{F}_{P, \alpha+\alpha_{\Delta} / 3} \mid S\right)\right\}
\end{aligned}
$$

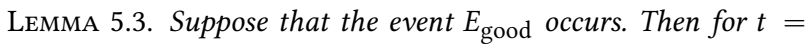
$t_{\text {final }}+1$, the set $\mathcal{M}_{\alpha_{t}-\alpha_{\Delta} / 2, t}$ is nonempty.

Proof. Let $v$ be a node as guaranteed by Lemma 5.1, i.e., so that $\hat{\mathcal{G}}\left(\alpha_{t}-\alpha_{\Delta}, v\right)$ is $k_{t}$-irreducible, and so that $\operatorname{Ldim}\left(\hat{\mathcal{G}}\left(\alpha_{t}-\alpha_{\Delta}, v\right)\right)=$ $\operatorname{Ldim}\left(\hat{\mathcal{G}}\left(\alpha_{t}, v\right)\right) \geqslant 0$. Since the event $E_{\text {good }}$ holds,

$$
\begin{aligned}
& \hat{\mathcal{G}}\left(\alpha_{t}-\alpha_{\Delta}, v\right)=\left.\left.\mathcal{F}_{\hat{P}_{S_{n}}, \alpha_{t}-\alpha_{\Delta}}\right|_{\mathbf{a}(v)} \subset \mathcal{F}_{P, \alpha_{t}-5 \alpha_{\Delta} / 6}\right|_{\mathbf{a}(v)} \\
& \left.\left.\subset \mathcal{F}_{P, \alpha_{t}-\alpha_{\Delta} / 6}\right|_{\mathbf{a}(v)} \subset \mathcal{F}_{\hat{P}_{S_{n}}, \alpha_{t}}\right|_{\mathbf{a}(v)}=\hat{\mathcal{G}}\left(\alpha_{t}, v\right) .
\end{aligned}
$$

It follows from Lemma 4.1 that $\left.\mathcal{F}_{P, \alpha_{t}-5 \alpha_{\Delta} / 6}\right|_{\mathbf{a}(v)}$ is $k_{t}$-irreducible and that $\operatorname{Ldim}\left(\left.\mathcal{F}_{P, \alpha_{t}-\alpha_{\Delta} / 6}\right|_{\mathbf{a}(v)}\right)=\operatorname{Ldim}\left(\left.\mathcal{F}_{P, \alpha_{t}-5 \alpha_{\Delta} / 6}\right|_{\mathbf{a}(v)}\right)$. Since the height of the tree $\hat{\mathbf{x}}^{(t-1)}=\hat{\mathbf{x}}^{\left(t_{\text {final }}\right)}$ is at most $k_{t}-k^{\prime}$ (Lemma 5.2), it follows that the number of tuples in $\mathbf{a}(v)$ is at most $k_{t}-k^{\prime}$; thus, after duplicating some of the tuples in a $(v)$ if necessary, we get that $\mathbf{a}(v) \in \mathcal{M}_{\alpha-\alpha_{\Delta} / 2, t}$.

For any $\alpha \in[0,1], t \in[d+1]$ for which $\mathcal{M}_{\alpha, t}$ is nonempty, define:

$$
\begin{aligned}
& S_{\alpha, t}^{\star} \in \underset{S \in \mathcal{M}_{\alpha, t}}{\arg \max }\left\{\operatorname{Ldim}\left(\left.\mathcal{F}_{P, \alpha}\right|_{S}\right)\right\}, \\
& \ell_{\alpha, t}^{\star}:=\max _{S \in \mathcal{M}_{\alpha, t}}\left\{\operatorname{Ldim}\left(\left.\mathcal{F}_{P, \alpha}\right|_{S}\right)\right\} \geqslant 0 .
\end{aligned}
$$

Also set

$$
\sigma_{\alpha, t}^{\star}:=\mathrm{SOA}_{\left.\mathcal{F}_{P, \alpha}\right|_{S_{\alpha, t}^{\star}}} .
$$

We emphasize here that $\mathcal{M}_{\alpha, t}$ and $S_{\alpha, t}^{\star}$ are both independent of the output of the algorithm ReduceTree (and in particular, they do not depend on the particular input dataset $S_{n}$ ).

LEMmA 5.4. Under the event $E_{\text {good, }}$, the following holds: for $t=$ $t_{\text {final }}+1 \in[d+1]$ and some leaf $\hat{v} \in \hat{\mathcal{L}}^{\prime}$, we have $\sigma_{\alpha_{t}-\alpha_{\Delta} / 2, t}^{\star}=$ $\mathrm{SOA}_{\hat{\mathcal{G}}\left(\alpha_{t}-2 \alpha_{\Delta} / 3, \hat{v}\right)}$. (In particular, for this $t, \sigma_{\alpha_{t}-\alpha_{\Delta} / 2, t}^{\star}$ is well-defined, i.e., $\mathcal{M}_{\alpha_{t}-\alpha_{\Delta} / 2, t}$ is nonempty.)

Moreover, $\hat{\mathcal{G}}\left(\alpha_{t}-2 \alpha_{\Delta} / 3, \hat{v}\right)$ is $k^{\prime}$-irreducible and nonempty, and $\operatorname{Ldim}\left(\hat{\mathcal{G}}\left(\alpha_{t}-2 \alpha_{\Delta} / 3, \hat{v}\right)\right)=\ell_{\alpha_{t}-\alpha_{\Delta} / 2, t}^{\star} \geqslant 0$.
Lemma 5.5. The set $\hat{\mathcal{S}}$ output by ReduceTree has size $|\hat{\mathcal{S}}| \leqslant$ $\prod_{t=1}^{d}\left(k_{t}+1\right)$.

The proofs of Lemmas 5.4 and 5.5 may be found in the full version of this paper [34].

\subsection{Building Block: Sparse Selection Protocol}

We use the following primitive for solving the sparse selection problem from [35]:

Definition 5.1 (Sparse selection). For $m, \ell \in \mathbb{N}$, in $(m, \ell)$-sparse selection problem, there is some (possibly infinite) universe $\mathcal{U}$, and $m$ users. Each user $i \in[m]$ is given some set $\mathcal{S}_{i} \subset \mathcal{U}$ of size $\left|\mathcal{S}_{i}\right| \leqslant \ell$. An algorithm solves the $(m, \ell)$-sparse selection problem with with additive error $\eta$ if it outputs some universe element $\hat{u} \in \mathcal{U}$ such that

$$
\left|\left\{i: \hat{u} \in \mathcal{S}_{i}\right\}\right| \geqslant \max _{u \in \mathcal{U}}\left|\left\{i: u \in \mathcal{S}_{i}\right\}\right|-\eta .
$$

Proposition 5.6 shows that the sparse selection problem can be solved privately with error independent of the size of the universe $\mathcal{U}$. It can be thought of as an analogue of the private stable histogram of [22, Proposition 2.20] for the problem of private selection.

Proposition 5.6 ([35], Lemma 36). For $\varepsilon \in(0,1], \delta \in(0,1)$, $\beta \in(0,1)$, there is an $(\varepsilon, \delta)$-differentially private algorithm that given an input dataset to the $(m, \ell)$-sparse selection problem, outputs a universe element $\hat{u}$ such that with probability at least $1-\beta$, the error of $\hat{u}$ is

$$
O\left(\frac{1}{\varepsilon} \log \left(\frac{m \ell}{\varepsilon \delta \beta}\right)\right) .
$$

\subsection{Overall Algorithm}

In this section we combine the components of Sections 5.1 and 5.2 to prove the following theorem, which gives an improper learner for hypothesis classes with sample complexity polynomial in the Littlestone dimension.

Theorem 5.7. Let $\mathcal{F}$ be a concept class of domain $\mathcal{X}$ with $d_{\mathrm{V}}:=$ $\operatorname{VCdim}(\mathcal{F}), d_{\mathrm{L}}:=\operatorname{Ldim}(\mathcal{F})$. For any $\varepsilon, \delta, \eta \in(0,1)$, for some

$$
n=O\left(\frac{d_{\mathrm{L}}^{5} d_{\mathrm{V}} \log ^{2}\left(\frac{d_{\mathrm{L}}}{\varepsilon \delta \eta \beta}\right)}{\varepsilon \eta^{2}}\right)
$$

the algorithm PolyPriLearn (Algorithm 2) takes as input n i.i.d. samples from any realizable distribution $P$, is $(\varepsilon, \delta)$-differentially private, and produces a hypothesis $\hat{f}$ so that $\operatorname{err}_{P}(\hat{f}) \leqslant \eta$ with probability at least $1-\beta$.

Moreover, under the same $(1-\beta)$-probability event, $\hat{f}=\mathrm{SOA}_{\mathcal{G}}$ for some $\mathcal{G} \subset \mathcal{F}$ for which $\mathcal{G}$ is $\left\lceil\frac{64 C_{0} d_{\mathrm{L}}}{\eta^{2}}\right\rceil$-irreducible.

Remark 5.1. The assertion that $\hat{f}=\mathrm{SOA}_{\mathcal{G}}$ for some $\mathcal{G}$ which is $\left\lceil\frac{64 C_{0} d_{\mathrm{L}}}{\eta^{2}}\right\rceil$-irreducible is for use in Section 6 when we use

PolyPriLearn as a component of a proper private learning algorithm.

PolyPriLearn (Algorithm 2) operates as follows. For sufficiently large positive integers $m, n_{0}$, PolyPriLearn runs ReduceTree on $m$ independent samples of size $n_{0}$ from the distribution $P$. Each 
run of ReduceTree outputs some set $\hat{\mathcal{S}}$ of classifiers in $\{-1,1\}^{X}$. PolyPriLearn then uses the sparse selection protocol of Proposition 5.6 to choose some classifier that lies in many of the sets $\hat{\mathcal{S}}$.

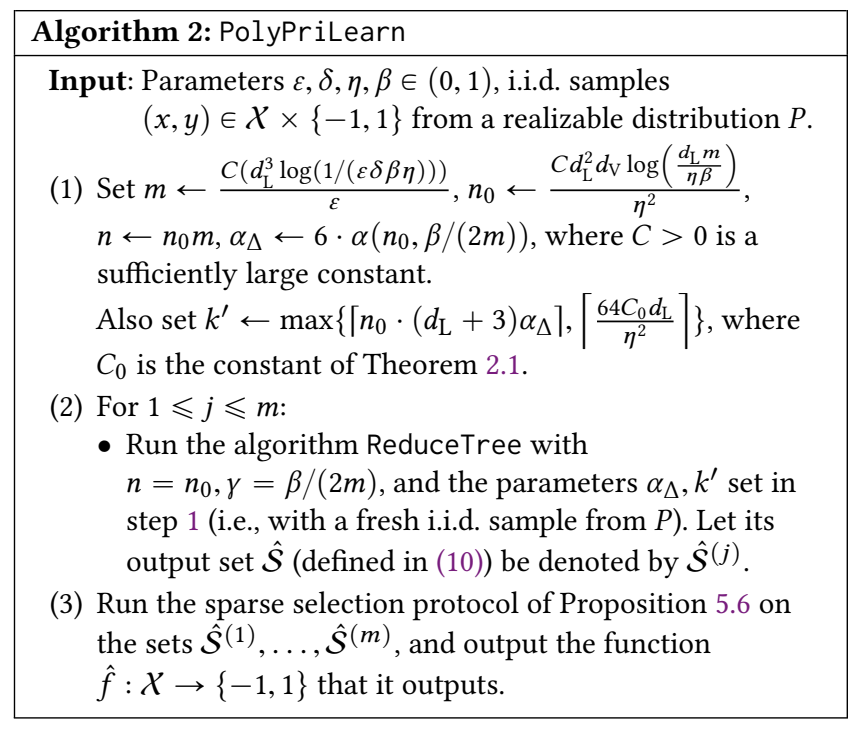

Proof of Theorem 5.7. In the proof we will often refer to the parameters $n_{0}, m, \alpha_{\Delta}, k^{\prime}$, which are set in step 1 of PolyPriLearn (Algorithm 2). Notice that by our choice of

$$
n_{0}=\frac{C d_{\mathrm{L}}^{2} d_{\mathrm{V}} \log \left(\frac{d_{\mathrm{L}} m}{\eta \beta}\right)}{\eta^{2}},
$$

as long as $C$ is sufficiently large, we have that $\alpha_{\Delta}:=6 \cdot \alpha\left(n_{0}, \beta /(2 m)\right)$ satisfies $\left(d_{\mathrm{L}}+3\right) \cdot \alpha_{\Delta}<\eta$. Recall the definition of $\alpha_{t}:=\left(d_{\mathrm{L}}+3-\right.$ $t) \cdot \alpha_{\Delta}$ for $1 \leqslant t \leqslant d_{\mathrm{L}}+1$ from ReduceTree.

For $1 \leqslant j \leqslant m$, let $T^{(j)}:=\left\{\left(x_{1}^{(j)}, y_{1}^{(j)}\right), \ldots,\left(x_{n_{0}}^{(j)}, y_{n_{0}}^{(j)}\right)\right\}$ be the dataset of size $n_{0}$ drawn (i.i.d. from $P$ ) in the $j$ th iteration of Step 2 of PolyPriLearn. Let $\hat{P}^{(j)}:=\frac{1}{n_{0}} \sum_{i=1}^{n_{0}} \delta_{\left(x_{i}^{(j)}, y_{i}^{(j)}\right)}$ be the empirical measure over $T^{(j)}$.

We say that a class $\mathcal{G} \subset \mathcal{F}$ is a finite restriction subclass (of $\mathcal{F}$ ) if we can write $\mathcal{G}=\left.\mathcal{F}\right|_{\left(x_{1}, y_{1}\right), \ldots,\left(x_{M}, y_{M}\right)}$ for some $\left(x_{1}, y_{1}\right), \ldots$, $\left(x_{M}, y_{M}\right) \in \mathcal{X} \times\{-1,1\}$. Note that the set of all finite restriction subclasses of $\mathcal{F}$ is countable by our assumption that $X$ is countable. It follows that the set of all finite unions of finite restriction subclasses of $\mathcal{F}$ is also countable. Now define

$\tilde{\mathcal{F}}=\mathcal{F} \cup\left\{\operatorname{SOA}_{\mathcal{G}}: \begin{array}{c}\mathcal{G} \subset \mathcal{F}, \mathcal{G} \text { is nonempty, }\left(d_{\mathrm{L}}+1\right) \text {-irreducible, } \\ \text { and a finite union of finite restriction subclasses of } \mathcal{F}\end{array}\right\}$.

Notice that the set $\hat{\mathcal{S}}$ output by ReduceTree consists entirely of functions in $\tilde{\mathcal{F}}$. (This follows since the set $\hat{\mathcal{S}}$ consists of hypotheses of the form $\operatorname{SOA}_{\hat{\mathcal{G}}(\alpha, v)}$, where $\hat{\mathcal{G}}(\alpha, v)$ is $k^{\prime}$-irreducible: we then use that $d_{\mathrm{L}}+1 \leqslant k^{\prime}$ and that for any $\alpha \in[0,1]$, and any dataset $S_{n}$, $\mathcal{F}_{\hat{P}_{S_{n}, \alpha}}$ is the union of at most $2^{n}$ finite restriction subclasses of $\mathcal{F}$.) Moreover, $\tilde{\mathcal{F}}$ is countable, and Lemma 4.3 gives that $\operatorname{VCdim}(\tilde{\mathcal{F}}) \leqslant$
$\operatorname{Ldim}(\tilde{\mathcal{F}}) \leqslant d_{\mathrm{L}}$. Then Theorem 2.1 gives that

$$
\begin{aligned}
& \operatorname{Pr}\left[\forall j \in[m]: \sup _{\tilde{f} \in \tilde{\mathcal{F}}}\left|\operatorname{err}_{P}(\tilde{f})-\operatorname{err}_{\hat{P}^{(j)}}(\tilde{f})\right| \leqslant \frac{\alpha_{\Delta}}{6}\right] \\
\geqslant & 1-(\beta /(2 m)) \cdot m=1-\beta / 2 .
\end{aligned}
$$

Let $E_{0}$ be the event inside the probability above, namely that for all $j \in[m], \sup _{\tilde{f} \in \tilde{\mathcal{F}}}\left|\operatorname{err}_{P}(\tilde{f})-\operatorname{err}_{\hat{P}^{(j)}}(\tilde{f})\right| \leqslant \frac{\alpha_{\Delta}}{6}$. Since $\tilde{\mathcal{F}} \supseteq \mathcal{F}, E_{0}$ contains the event that $E_{\text {good }}$ simultaneously holds for each dataset $T^{(1)}, \ldots, T^{(m)}$ (recall that $E_{\text {good }}$ was defined for any dataset $T^{(j)}$ in (8)).

The bulk of the proof of Theorem 5.7 is to show the following two claims:

CLAIm 5.8. Suppose $m>\frac{C d_{\mathrm{L}}^{3} \log \left(\frac{1}{\varepsilon \delta \beta \eta}\right)}{\varepsilon}$ for a sufficiently large constant $C>0$. There is an event $E_{1}$ that occurs with probability at least $1-\beta / 2$ (over the randomness of the dataset and the algorithm), so that under $E_{1} \cap E_{0}$, PolyPriLearn outputs a hypothesis $\mathrm{SOA}_{\mathcal{G}}$, for some $\mathcal{G} \subset \mathcal{F}$ so that $\mathcal{G}$ is $k^{\prime}$-irreducible. Moreover, this hypothesis belongs to $\hat{\mathcal{S}}^{(j)}$ for some $j \in[m]$.

Claim 5.9. Suppose $k^{\prime} \geqslant\left\lceil n_{0} \cdot\left(d_{\mathrm{L}}+2\right) \cdot \alpha_{\Delta}\right\rceil$. Under the event $E_{1} \cap E_{0}$, the output of PolyPriLearn has empirical error at most $\left(d_{\mathrm{L}}+2\right) \cdot \alpha_{\Delta}$ on at least one of the $m$ datasets $T^{(j)}$ drawn in Step 2 of PolyPriLearn.

Assuming Claims 5.8 and 5.9, we complete the proof of Theorem 5.7. Notice that the assumptions of Claims 5.8 and 5.9 hold by our choices of $m, k^{\prime}$ in Step 1 of PolyPriLearn. Denote the output of PolyPriLearn by $\hat{f}: \mathcal{X} \rightarrow\{-1,1\}$. By Claim 5.9, we have that $\operatorname{err}_{\hat{P}^{(j)}}(\hat{f}) \leqslant\left(d_{\mathrm{L}}+2\right) \cdot \alpha_{\Delta}$ for some $j \in[m]$. By Claim 5.8 and the definition of the sets $\hat{\mathcal{S}}^{(j)}$ in (10), we have that under the event $E_{1} \cap E_{0}$, $\hat{f} \in \tilde{\mathcal{F}}$; moreover, $\hat{f}=\mathrm{SOA}_{\mathcal{G}}$ for some $\mathcal{G} \subset \mathcal{F}$ which is $\left\lceil\frac{64 C_{0} d_{\mathrm{L}}}{\eta^{2}}\right\rceil$ irreducible, by the choice of $k^{\prime}$ in step 1 of Algorithm 2. By the definition of $E_{0}$, it follows that under the event $E_{0} \cap E_{1}$, since $\hat{f} \in \tilde{\mathcal{F}}$, we have $\operatorname{err}_{P}(\hat{f}) \leqslant\left(d_{\mathrm{L}}+2\right) \cdot \alpha_{\Delta}+\alpha_{\Delta} / 6 \leqslant\left(d_{\mathrm{L}}+3\right) \cdot \alpha_{\Delta} \leqslant \eta$. By (15) and a union bound, $\operatorname{Pr}\left[E_{0} \cap E_{1}\right] \geqslant 1-\beta$, so $\operatorname{Pr}\left[\operatorname{err}_{P}(\hat{f}) \leqslant \eta\right] \geqslant 1-\beta$, as desired.

That PolyPriLearn is $(\varepsilon, \delta)$-differentially private follows as an immediate consequence of Proposition 5.6 and the fact that each data point lies in exactly one $T^{(j)}$. Summarizing, the sample complexity of PolyPriLearn is

$$
n_{0} \cdot m \leqslant O\left(\frac{d_{\mathrm{L}}^{5} d_{\mathrm{V}} \log ^{2}\left(\frac{d_{\mathrm{L}}}{\varepsilon \delta \eta \beta}\right)}{\varepsilon \eta^{2}}\right)
$$

Finally we prove Claims 5.8 and 5.9.

Proof of Claim 5.8. Notice that for each $j \in[m]$, each element of $\hat{\mathcal{S}}^{(j)}$ is of the form $\mathrm{SOA}_{\mathcal{G}}$ for some $\mathcal{G} \subset \mathcal{F}$ which is $k^{\prime}$-irreducible, and thus $\left(d_{\mathrm{L}}+1\right)$-irreducible (as $\left.k^{\prime} \geqslant d_{\mathrm{L}}+1\right)$. It therefore suffices to show that under the event $E_{0} \cap E_{1}$ (for an appropriate choice of $E_{1}$ ), PolyPriLearn outputs some element of some $\hat{\mathcal{S}}^{(j)}, j \in[m]$.

For $\alpha \in[0,1], t \in\left[d_{\mathrm{L}}+1\right]$, recall the definition $\mathcal{M}_{\alpha, t}$ in (11), and for those $\alpha, t$ for which $\mathcal{M}_{\alpha, t}$ is nonempty, the definition of $\sigma_{\alpha, t}^{\star}$ 
in (13). By the definition of $\hat{\mathcal{S}}^{(j)}$ (see (10)) and Lemma 5.4, under the event $E_{0}$ each $\hat{\mathcal{S}}^{(j)}$ contains at least one of $\sigma_{\alpha_{t}-\alpha_{\Delta} / 2, t}^{\star}$ for some $t \in\left[d_{\mathrm{L}}+1\right]$ (which is well-defined). By the pigeonhole principle, it follows that some $\sigma_{\alpha_{t}-\alpha_{\Delta} / 2, t}^{\star}$ lies in at least $\left[m /\left(d_{\mathrm{L}}+1\right)\right\rceil$ sets $\hat{\mathcal{S}}^{(j)}$.

By Lemma 5.5, we have that

$\left|\hat{\mathcal{S}}^{(j)}\right| \leqslant \prod_{t=1}^{d_{\mathrm{L}}}\left(k_{t}+1\right)=\prod_{t=1}^{d_{\mathrm{L}}} k^{\prime} \cdot 2^{t}=\left(k^{\prime}\right)^{d_{\mathrm{L}}} \cdot 2^{\left(d_{\mathrm{L}}+1\right) d_{\mathrm{L}} / 2} \leqslant 2^{d_{\mathrm{L}}^{2}+d_{\mathrm{L}} \log k^{\prime}}$

Now choose $v>0$ so that the $\left(m, 2^{d_{\mathrm{L}}^{2}+d_{\mathrm{L}} \log k^{\prime}}\right)$-sparse selection protocol of Proposition 5.6 (with universe $\mathcal{U}=\tilde{\mathcal{F}}$ ), has error at most $v$ on some event $E_{1}$ with probability at least $1-\beta / 2$. By Proposition 5.6, we may choose $v=\frac{C}{\varepsilon} \log \left(\frac{m 2^{d_{\mathrm{L}}^{2}+d_{\mathrm{L}} \log k^{\prime}}}{\varepsilon \delta \beta}\right)$ for a sufficiently large constant $C$.

Summarizing, under the event $E_{0} \cap E_{1}$, as long as $v<\left\lceil m /\left(d_{\mathrm{L}}+\right.\right.$ $1)]$, the hypothesis $\hat{f}$ output by the sparse selection protocol belongs to some set $\hat{\mathcal{S}}^{(j)}$. Since

$$
\begin{aligned}
k^{\prime} & \leqslant 200 C_{0} \max \left\{n_{0}\left(d_{\mathrm{L}}+3\right) \alpha_{\Delta}, \frac{d_{\mathrm{L}}}{\eta^{2}}\right\} \leqslant 200 C_{0} \max \left\{\frac{d_{\mathrm{L}}}{\eta^{2}}, n_{0} \eta\right\} \\
& \leqslant \frac{200 C_{0} d_{\mathrm{L}}^{2} d_{\mathrm{V}} \log \left(\frac{d_{\mathrm{L}} m}{\eta \beta}\right)}{\eta^{2}}
\end{aligned}
$$

to ensure $v<\left\lceil m /\left(d_{\mathrm{L}}+1\right)\right]$ it suffices to have

$$
\begin{array}{r}
m>\frac{C^{\prime}\left(d_{\mathrm{L}}+1\right)}{\varepsilon}\left(\log (m)+d_{\mathrm{L}}^{2}+\log \left(\frac{1}{\varepsilon \delta \beta}\right)\right. \\
\left.+d_{\mathrm{L}}\left(\log (1 / \eta)+\log \log \left(\frac{m}{\beta}\right)\right)\right),
\end{array}
$$

for which it in turn suffices that $m \geqslant \frac{C^{\prime \prime} d_{\mathrm{L}}^{3} \log \left(\frac{1}{\varepsilon \delta \beta \eta}\right)}{\varepsilon}$ for sufficiently large constants $C^{\prime}, C^{\prime \prime}$.

Proof of Claim 5.9. By Claim 5.8, it suffices to show that under the event $E_{1} \cap E_{0}$, each element of $\hat{\mathcal{S}}^{(j)}$ has empirical error at most $\left(d_{\mathrm{L}}+2\right) \cdot \alpha_{\Delta}$ on the dataset $T^{(j)}$. By definition, each element

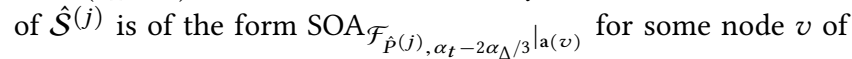
the tree $\hat{\mathbf{x}}$ output by ReduceTree for which $\mathcal{F}_{\hat{P}^{(j)}, \alpha_{t}-2 \alpha_{\Delta} /\left.3\right|_{\mathbf{a}(v)}}$ is nonempty and $k^{\prime}$-irreducible (see (10)). Fix any such element, and write $\hat{\mathcal{H}}:=\left.\mathcal{F}_{\hat{P}^{(j)}, \alpha_{t}-2 \alpha_{\Delta} / 3}\right|_{\mathbf{a}(v)}$. By definition we have that each $f \in \hat{\mathcal{H}}=\left.\mathcal{F}_{\hat{P}^{(j)}, \alpha_{t}-2 \alpha_{\Delta} / 3}\right|_{\mathbf{a}(v)} \subset \mathcal{F}_{\hat{P}^{(j)}, \alpha_{t}-2 \alpha_{\Delta} / 3}$ satisfies

$\operatorname{err}_{\hat{P}^{(j)}}(f) \leqslant \alpha_{t}-2 \alpha_{\Delta} / 3 \leqslant \alpha_{1}-2 \alpha_{\Delta} / 3=\left(d_{\mathrm{L}}+2\right) \cdot \alpha_{\Delta}-2 \alpha_{\Delta} / 3$

Let $\ell=\left\lceil n_{0} \alpha_{\Delta} \cdot\left(d_{\mathrm{L}}+2\right)\right\rceil$. Suppose for the purpose of contradiction that

$$
\operatorname{err}_{\hat{P}^{(j)}}\left(\operatorname{SOA}_{\hat{\mathcal{H}}}\right) \geqslant \alpha_{\Delta} \cdot\left(d_{\mathrm{L}}+2\right) .
$$

Let $i_{1}, \ldots, i_{\ell} \in\left[n_{0}\right]$ be indices on which $\mathrm{SOA}_{\hat{\mathcal{H}}}$ is incorrect; i.e., for $t \in[\ell]$, we have $\operatorname{SOA}_{\hat{\mathcal{H}}}\left(x_{i_{t}}^{(j)}\right)=-y_{i_{t}}^{(j)}$, i.e., $\operatorname{Ldim}\left(\left.\hat{\mathcal{H}}\right|_{\left(x_{i_{t}}^{(j)},-y_{i_{t}}^{(j)}\right)}\right)=$ $\operatorname{Ldim}(\hat{\mathcal{H}})$. Since $\hat{\mathcal{H}}$ is $k^{\prime}$-irreducible and $k^{\prime} \geqslant \ell$, it follows that

$$
\operatorname{Ldim}\left(\left.\hat{\mathcal{H}}\right|_{\left(x_{i_{1}},-y_{i_{1}}^{(j)}\right), \ldots,\left(x_{i_{\ell}}^{(j)},-y_{i_{\ell}}^{(j)}\right)}\right)=\operatorname{Ldim}(\hat{\mathcal{H}}),
$$

and in particular since $\hat{\mathcal{H}}$ is nonempty there is some $f \in \hat{\mathcal{H}}$ so that for $t \in[\ell], f\left(x_{i_{t}}^{(j)}\right)=-y_{i_{t}}^{(j)}$, i.e., $\operatorname{err}_{\hat{P}^{(j)}}(f) \geqslant \ell / n_{0}>\alpha_{\Delta} \cdot\left(d_{\mathrm{L}}+\right.$ 2) $-2 \alpha_{\Delta} / 3$. This is a contradiction to (16).

This concludes the proof of Theorem 5.7.

\section{PROPER PRIVATE LEARNER FOR LITTLESTONE CLASSES}

In this section we show how to use the improper private learner of Theorem 5.7 to obtain a proper one, thus proving Theorem 6.3 (the formal version of Theorem 1.1). For simplicity we assume in this section that $\mathcal{X}, \mathcal{F}$ are finite. The case in which they are allowed to be infinite is treated in the appendix of the full version of this paper [34]. Let $\Delta(\mathcal{X}), \Delta(\mathcal{F})$ be the spaces of probability distributions over $\mathcal{X}, \mathcal{F}$, respectively.

Lemma 6.1. Let $C_{0}$ be the constant of Theorem 2.1. Fix any $\alpha \in$ $(0,1)$ and $\mathcal{G} \subset \mathcal{F}$ which is $\left\lceil\frac{C_{0} d}{\alpha^{2}}\right\rceil$-irreducible and suppose

$\operatorname{VCdim}(\mathcal{F}) \leqslant d$ and $\operatorname{VCdim}^{\star}(\mathcal{F}) \leqslant d^{\star}$ for some $d, d^{\star} \in \mathbb{N}$. Then there is a set $\mathcal{H} \subset \mathcal{F}$, depending only on the function $\mathrm{SOA}_{\mathcal{G}}: X \rightarrow$ $\{-1,1\}$, and of size $|\mathcal{H}| \leqslant\left\lceil\frac{C_{0} d^{\star}}{\alpha^{2}}\right\rceil$, so that for any distribution $P \in$ $\Delta(\mathcal{X})$, it holds that

$$
\min _{h \in \mathcal{H}} \mathbb{E}_{X \sim P}\left[1\left[h(x) \neq \operatorname{SOA}_{\mathcal{G}}(x)\right]\right] \leqslant 2 \alpha .
$$

The proof of Lemma 6.1 proceeds by using von Neumann's minimax theorem and uniform convergence (i.e., Theorem 2.1); it can be found in the full version of this paper [34].

\subsection{Private Proper Learning Protocol}

Before introducing our private proper learning algorithm, we need the following basic lemma which establishes that the use of the exponential mechanism can output a good hypothesis privately from a class of small size:

Lemma 6.2 (Generic Private Learner, [43]). Let $\mathcal{H} \subset\{-1,1\}^{X}$ be a finite set of hypotheses. For

$$
n=O\left(\frac{\log |\mathcal{H}|+\log 1 / \beta}{\alpha \varepsilon}\right),
$$

there is an $(\varepsilon, 0)$-differentially private algorithm GenericLearner: $(\mathcal{X} \times\{-1,1\})^{n} \rightarrow \mathcal{H}$ so that the following holds. For any distribution $P$ over $\mathcal{X} \times\{-1,1\}$ so that there exists $h^{\star} \in \mathcal{H}$ with

$$
\operatorname{err}_{P}\left(h^{\star}\right) \leqslant \alpha,
$$

on input $S_{n}:=\left\{\left(x_{1}, y_{1}\right), \ldots,\left(x_{n}, y_{n}\right)\right\} \sim P^{n}$, GenericLearner outputs, with probability at least $1-\beta$, a hypothesis $\hat{h} \in \mathcal{H}$ so that

$$
\operatorname{err}_{P}(\hat{h}) \leqslant 2 \alpha .
$$
16].

The precise formulation of Lemma 6.2 is proved in [21, Lemma

Our algorithm, PolyPriPropLearn, for privately and properly learning a hypothesis class, is presented in Algorithm 3. Given sufficiently many samples from a realizable distribution $P$,

PolyPriPropLearn first runs PolyPriLearn to come up with a hypothesis of the form $\mathrm{SOA}_{\mathcal{G}} \in\{-1,1\}^{X}$ with low population loss on the distribution $P$. It then uses the guarantee of Lemma 6.1 to come up with a small subclass $\mathcal{H} \subset \mathcal{F}$ which is guaranteed to 
contain a hypothesis that performs nearly as well as $\mathrm{SOA}_{\mathcal{G}}$ on the distribution $P$. It then privately chooses such a hypothesis $\hat{h} \in \mathcal{H}$ using the exponential mechanism (Lemma 6.2).

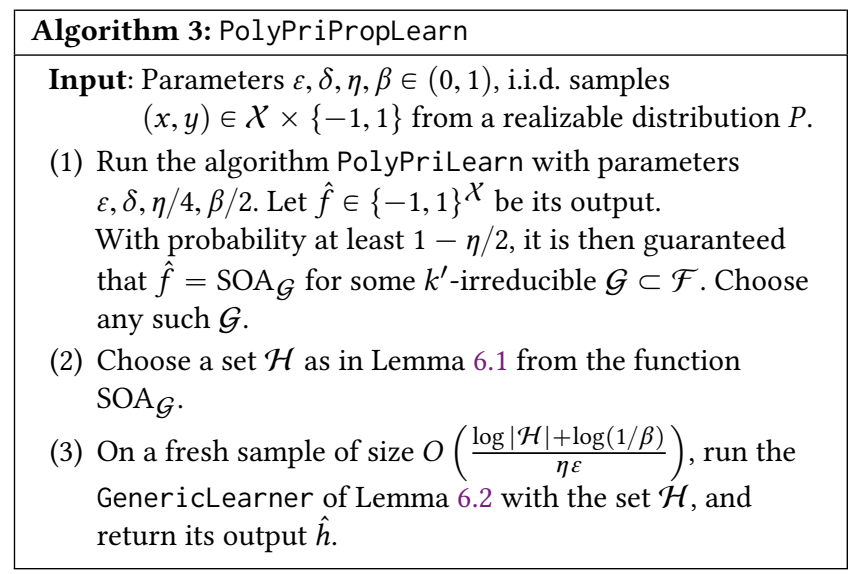

Theorem 6.3 (Private Proper PAC LeArning). Let $\mathcal{F}$ be a concept class of domain $\mathcal{X}$ with $d_{\mathrm{V}}:=\operatorname{VCdim}(\mathcal{F}), d_{\mathrm{L}}:=\operatorname{Ldim}(\mathcal{F})$. For any $\varepsilon, \delta, \eta, \beta \in(0,1)$, for some

$$
n=O\left(\frac{d_{\mathrm{L}}^{5} d_{\mathrm{V}} \log ^{2}\left(\frac{d_{\mathrm{L}}}{\varepsilon \delta \eta \beta}\right)}{\varepsilon \eta^{2}}\right) \text {, }
$$

there is an $(\varepsilon, \delta)$-differentially private algorithm $A:(X \times\{-1,1\})^{n} \rightarrow$ $\{-1,1\}^{\mathcal{X}}$, which, given $n$ i.i.d. samples from any realizable distribution $P$, products a hypothesis $\hat{f}$ so that $\operatorname{err}_{P}(\hat{f}) \leqslant \eta$ with probability at least $1-\beta$.

The proof of Theorem 6.3 may be found in the full version of this paper [34]. As a corollary of Theorem 6.3 and [10, Theorem 4.16] (or [3, Theorem 2.4], which is a more general result) we get a sample complexity bound for agnostic private proper PAC learning:

Corollary 6.4 (Agnostic Private Proper PAC learning). Let $\mathcal{F}$ be a concept class of domain $\mathcal{X}$ with $d_{\mathrm{V}}:=\operatorname{VCdim}(\mathcal{F}), d_{\mathrm{L}}:=$ $\operatorname{Ldim}(\mathcal{F})$. For any $\varepsilon, \delta, \eta, \beta \in(0,1)$, for some

$$
n=O\left(\frac{d_{\mathrm{L}}^{5} d_{\mathrm{V}} \log ^{2}\left(\frac{d_{\mathrm{L}}}{\varepsilon \delta \eta \beta}\right)}{\varepsilon \eta^{2}}\right),
$$

there is an $(\varepsilon, \delta)$-differentially private algorithm $A:(X \times\{-1,1\})^{n} \rightarrow$ $\mathcal{F}$, which, given $n$ i.i.d. samples from any distribution $P$, produces $a$ hypothesis $\hat{f} \in \mathcal{F}$ so that $\operatorname{err}_{P}(\hat{f}) \leqslant \eta+\inf _{f \in \mathcal{F}} \operatorname{err}_{P}(f)$ with probability at least $1-\beta$.

\subsection{Application to Private Data Sanitization}

In this section we show how to prove Corollaries 1.2 and 1.3 using a result of [18] that shows how to convert a private proper agnostic PAC learner into a sanitizer for a binary hypothesis class. ${ }^{8} \mathrm{We}$ say that an algorithm $A$ is an $(\alpha, \beta)$-accurate proper agnostic PAC learner for a class $\mathcal{F}$ with sample complexity $n$ if for any distribution

${ }^{8}$ We remark that the results of [18] do not require the assumption of countability on $\mathcal{F}, \mathcal{X}$ that we make in this paper.
$P$ over $X \times\{-1,1\}$, when given as input $n$ i.i.d. samples from $P$, the algorithm $A$ produces as output a function $\hat{f} \in \mathcal{F}$ so that with probability at least $1-\beta$ over the sample and the randomness in $A$, we have $\operatorname{err}_{P}(\hat{f}) \leqslant \alpha+\inf _{f \in \mathcal{F}} \operatorname{err}_{P}(f)$.

Theorem 6.5 (Slight strengthening of [18], Propositions 1 ¿ 2). Suppose $\mathcal{F} \subset\{-1,1\}^{X}$ is a class of VC dimension $d_{\mathrm{V}}$ and dual Littlestone dimension $d_{\mathrm{L}}^{\star}$. Moreover suppose that for any $\alpha^{\prime}, \beta^{\prime}, \varepsilon^{\prime}, \delta^{\prime} \in$ $(0,1)$, there is some $n_{0}\left(\alpha^{\prime}, \beta^{\prime}, \varepsilon^{\prime}, \delta^{\prime}\right) \in \mathbb{N}$ so that $\mathcal{F}$ has a proper PAC learner with sample complexity $n_{0}\left(\alpha^{\prime}, \beta^{\prime}, \varepsilon^{\prime}, \delta^{\prime}\right)$ that is $\left(\varepsilon^{\prime}, \delta^{\prime}\right)$ differentially private and $\left(\alpha^{\prime}, \beta^{\prime}\right)$-accurate. Then there is a (sufficiently large) constant $C>0$ so that for any $\alpha, \beta, \varepsilon, \delta \in(0,1)$, as long as $n \in \mathbb{N}$ is chosen to satisfy

$$
n \geqslant \frac{C}{\varepsilon} \cdot\left(\left(n_{0}\left(\alpha / 8, \tau_{0} \beta / 2,1, \delta\right)+\frac{\log \left(\frac{d_{\mathrm{L}}^{\star}}{\beta \alpha}\right)}{\alpha}\right) \cdot\left(\frac{d_{\mathrm{L}}^{\star} \log \left(d_{\mathrm{L}}^{\star} / \alpha\right) \log (1 / \delta)}{\alpha^{2}}\right)^{1 / 2}\right)_{(19)}
$$

where $\tau_{0}=\frac{\alpha^{2}}{C d_{\mathrm{L}}^{\star} \log \left(d_{\mathrm{L}}^{\star} / \alpha\right) \log (1 / \delta)}$, it holds that $\mathcal{F}$ has a $\left(n, \alpha, \beta, 1, \delta \cdot \frac{\sqrt{C d_{\mathrm{L}}^{\star} \log \left(d_{\mathrm{L}}^{\star} / \alpha\right)}}{\alpha}\right)$-sanitizer.

The proof of Theorem 6.5 (using the proof technique in [18, Propositions $1 \& 2$ ]) is in the full version of this paper [34].

As an immediate corollary of Theorem 6.5 and Corollary 6.4 we obtain the following:

Corollary 6.6 (Private SANitization; Formal version of Corollary 1.2). Let $\mathcal{F}$ be a hypothesis class with VC dimension $d_{\mathrm{V}}$, Littlestone dimension $d_{\mathrm{L}}$, and dual Littlestone dimension $d_{\mathrm{L}}^{\star}$. For any $\alpha, \beta, \varepsilon, \delta \in(0,1)$, for any $n \in \mathbb{N}$ satisfying

$$
n \geqslant C \cdot \frac{d_{\mathrm{L}}^{5} d_{\mathrm{V}} \sqrt{d_{\mathrm{L}}^{\star}} \log ^{2}\left(\frac{d_{\mathrm{L}} d_{\mathrm{L}}^{\star}}{\delta \alpha \beta}\right) \log \left(\frac{d_{\mathrm{L}}^{\star}}{\alpha \delta}\right)}{\alpha^{3} \varepsilon},
$$

$\mathcal{F}$ has a $(n, \alpha, \beta, \varepsilon, \delta)$-sanitizer.

We remark that the dependence of $(20)$ on $d_{\mathrm{L}}^{\star}$, namely $\tilde{O}\left(\sqrt{d_{\mathrm{L}}^{\star}}\right)$, is tight up to polylogarithmic factors in the sense that for all $d_{\mathrm{L}}^{\star}$, there is a class $\mathcal{F}$ with $\max \{\operatorname{VCdim}(\mathcal{F}), \operatorname{Ldim}(\mathcal{F})\} \leqslant O\left(\log d_{\mathrm{L}}^{\star}\right)$ and $\operatorname{Ldim}^{\star}(\mathcal{F})=d_{\mathrm{L}}^{\star}$, yet the sample complexity of sanitization for $\mathcal{F}$ is $\tilde{\Omega}\left(\sqrt{d_{\mathrm{L}}^{\star}}\right)$, by Theorem 6.7 below.

The proof of Corollaries 6.6 and 1.3 may be found in the full version of this paper [34].

Lower bounds. We end this section by discussing how the sample complexity bound of Corollary 6.6 compares to existing lower bounds for sanitization. First, we remark that it follows from fingerprinting-based lower bounds [24] that in general the sample complexity of a sanitizer for a class $\mathcal{F}$ must grow at least polynomially in the the dual Littlestone dimension of $\mathcal{F}$ :

Theorem 6.7 ([24], Theorem 5.8). For any constant $\ell \in \mathbb{N}$, the following holds for all $d, t \in \mathbb{N}$ so that $\ell+2 \leqslant t \leqslant d / 2$. For $\mathcal{X}=$ $\{-1,1\}^{d}$, there is a class $\mathcal{F} \subset\{-1,1\}^{\mathcal{X}}$ so that:

- $\operatorname{Ldim}(\mathcal{F})=\Theta(t \log (d / t))$

- $\operatorname{Ldim}^{\star}(\mathcal{F})=\Theta(d)$, and so that for all $\varepsilon \in(0,1)$ and $\alpha \geqslant \tilde{\Omega}\left(\frac{d^{-\ell / 3+1 / 4}}{\sqrt{\varepsilon}}\right)$, any $(n, \alpha, 1 / 100, \varepsilon, 1 /(10 n))$-sanitizer for $\mathcal{F}$ must have $n \geqslant \tilde{\Omega}\left(\frac{t \sqrt{d}}{\varepsilon \alpha^{2}}\right)$. 
For any fixed $\ell$, the $\tilde{\Omega}(\cdot)$ in Theorem 6.7 hides factors which are inverse polynomial in $\log t, \log d, \log \frac{1}{\varepsilon}, \log \frac{1}{\alpha}$. We also remark that the VC and dual VC dimensions are within constant factors of the Littlestone and dual Littlestone dimensions of the class $\mathcal{F}$ of Theorem 6.7 (this will be clear from the proof below). By choosing $\ell=1$, and arbitrary positive integers $t, d$ tending to $\infty$ and satisfying $t \leqslant d / 2$, Theorem 6.7 rules out a sample complexity bound for sanitization that depends polynomially on only the Littlestone dimension of $\mathcal{F}$ (such as one in Theorem 6.3 for proper private learning). Because of the requirement that $t \leqslant d / 2$ in Theorem 6.7, it does not rule out a sample complexity bound that depends polynomially on only the dual Littlestone dimension (and only sub-polynomially on the Littlestone dimension). This latter possibility is ruled out by discrepancy-based lower bounds:

Theorem 6.8 ([47]). For any binary hypothesis class $\mathcal{F}$, any $\alpha<$ $1 / 50$ and any $\varepsilon \in(0,1)$, any $(n, \alpha, 1 / 100, \varepsilon, 0.1)$-sanitizer for $\mathcal{F}$ must have $n \geqslant \Omega\left(\frac{\operatorname{VCdim}(\mathcal{F})}{\varepsilon \alpha}\right)$.

For a proof of the precise statement of Theorem 6.8, see Theorem 5.8 and Proposition 5.11 of [54]. Note that for any positive integer $d$, there is a class $\mathcal{F}$ for which $\operatorname{Ldim}(\mathcal{F})=\operatorname{VCdim}(\mathcal{F})=d$ and $\operatorname{Ldim}^{\star}(\mathcal{F})=\Theta(\log d)$ (for instance, we may take the class of all functions on $d$ distinct points). Thus Theorem 6.8 rules out the existence of a sanitizer with sample complexity polynomial in only dual Littlestone dimension.

Summarizing, from Theorems 6.7 and 6.8, we obtain that Corollary 6.6 is "best possible up to a polynomial" in the sense that polynomial dependence on both $d_{\mathrm{L}}$ and $d_{\mathrm{L}}^{\star}$ is necessary in a worstcase sense. Moreover, when $d_{\mathrm{L}}, d_{\mathrm{L}}^{\star}$ are of the same order, then any sample complexity upper bound must be superlinear $\max \left\{d_{\mathrm{L}}, d_{\mathrm{L}}^{\star}\right\}$ (Theorem 6.7). Finally, in light of Theorem 6.7, the square-root dependence on $d_{\mathrm{L}}^{\star}$ (up to polylogarithmic factors) in Corollary 6.6 is best possible up to polylogarithmic factors.

\section{CONCLUSIONS}

In this paper we showed that it is possible to privately and properly learn binary hypothesis classes of Littlestone dimension $d$ with sample complexity polynomial in $d$. As a corollary we showed that such classes have sanitizers with sample complexity polynomial in $d$ and the dual Littlestone dimension $d^{\star}$. A central open question remaining (see, e.g., [11, Section 1.6]) is to determine a characterization of the sample complexity of (proper and improper) PAC learning with approximate differential privacy, up to (ideally) a constant factor, much like the VC dimension provides such a characterization for (non-private) PAC learning [55], the Littlestone dimension provides such a characterization for online learning [13, 44], and the probabilistic representation dimension [11] and the one-way public coin communication complexity [33] both provide such a characterization for improper PAC learning with pure differential privacy. As noted by [3], current lower bounds even allow for the possibility that the sample complexity of (proper or improper) PAC learning with approximate differential privacy is linear in $\operatorname{VCdim}(\mathcal{F})+\log ^{\star}(\operatorname{Ldim}(\mathcal{F}))$. Below we list some intermediate questions which may be useful in attacking this question and the related question of characterizing the sample complexity of sanitization. (Throughout by "private" we mean $(\varepsilon, \delta)$-differentially private with $\delta$ negligible in the number of users $n$.)

(1) Sample complexity linear in Littlestone dimension. The most immediate open question is to reduce the exponent of $d$ from the current value of 6 in Theorem 1.1. In particular, one could hope for sample complexity that scales linearly with the Littlestone dimension $d$ (see the discussion following Theorem 1.1).

(2) Polynomial characterization of private learnability. One could also attempt to show bounds with sublinear dependence on the Littlestone dimension, as long as there is at least linear dependence on the VC dimension. Rather optimistically, we ask: is the sample complexity of (properly or improperly) PAC learning a class $\mathcal{F}$ with $(\varepsilon, \delta)$-differential privacy at most $n=\operatorname{poly}\left(\operatorname{VCdim}(\mathcal{F}), \log ^{\star}(\operatorname{Ldim}(\mathcal{F}))\right)$ ? (Here we omit dependence on $1 / \alpha, 1 / \varepsilon, \log 1 / \delta$, for which the dependence should be polynomial as well.) In light of the lower bound of $\Omega\left(\operatorname{VCdim}(\mathcal{F})+\log ^{\star}(\operatorname{Ldim}(\mathcal{F}))\right)$ by Alon et al. [5] on the sample complexity, this would give a characterization for the sample complexity of private PAC learning up to a polynomial factor.

(3) Proper vs. improper learning. Is there a family of hypothesis classes for which the sample complexity of proper private learning is asymptotically larger than the sample complexity of improper private learning? The answer to this question is "yes" for the case of pure privacy (e.g., exhibited by the class of point functions [7]), but it remains open for approximate privacy to the best of our knowledge.

(4) Direct proof of Corollary 6.6. The current proof of Corollary 6.6 is quite long: it consists of first proving the existence of an improper private learner (Theorem 5.7), then showing how to make it proper (Corollary 6.4), and finally applying Theorem 6.5 of Bousquet et al. [18], which itself has two fairly involved parts, the first of which shows that $\mathcal{F}$ is "Sequentially-Foolable" [18, Theorem 2], and the second of which shows that $\mathcal{F}$ is sanitizable [18, Theorem 1]. It would be interesting to find a more direct proof of Corollary 6.6, namely one that does not "go through" a proper learner.

(5) Improved bounds for sanitization. Finally, it would be interesting to improve quantitatively upon the upper bound for sanitization of Corollary 6.6. In particular, analogously to item 2, it is natural to ask: is the sample complexity of sanitizating a class $\mathcal{F}$ (with approximate privacy) at most $n=\operatorname{poly}\left(\operatorname{VCdim}(\mathcal{F}), \operatorname{VCdim}^{\star}(\mathcal{F}), \log ^{\star}(\operatorname{Ldim}(\mathcal{F}))\right)$ ? By [24, Corollary 3.6], Theorem 6.8, and [23, Theorems 3.2 $\&$ 4.6], the sample complexity of sanitization is at least $\tilde{\Omega}\left(\operatorname{VCdim}(\mathcal{F})+\sqrt{\operatorname{VCdim}^{\star}(\mathcal{F})}+\log ^{\star}(\operatorname{Ldim}(\mathcal{F}))\right),{ }^{9}$ so

\footnotetext{
${ }^{9}[24$, Corollary 3.6] gives a lower bound of $\tilde{\Omega}(\sqrt{d})$ on the sample complexity of private release of 1-way marginals on $\{-1,1\}^{d}$; the $\tilde{\Omega}\left(\sqrt{\operatorname{VCdim}^{\star}(\mathcal{F})}\right)$ lower bound on the sample complexity of sanitization in any class $\mathcal{F}$ follows since a class of 1-way marginals on a copy of $\{-1,1\}^{\operatorname{VCdim}^{\star}(\mathcal{F})}$ may be embedded in any class $\mathcal{F}$. Similarly, [23] gives a $\Omega\left(\log ^{\star}|X|\right)$ lower bound on the sample complexity of release of threshold functions on a domain $\mathcal{X}$; the $\Omega\left(\log ^{\star}|\mathcal{X}|\right)$ lower bound on the sample complexity of sanitization in any class $\mathcal{F}$ follows since $\log \operatorname{Ldim}(\mathcal{F})$ thresholds may be embedded in $\mathcal{F}$
} 
this would provide a characterization for the sample complexity of sanitization up to a polynomial factor. Since our approach of using the results of [18] seems to necessarily incur at least a square-root dependence on the dual Littlestone $\operatorname{dimension} \operatorname{Ldim}^{\star}(\mathcal{F})$, any positive answer to this question would likely involve a positive answer to the question in item 4.

\section{ACKNOWLEDGEMENTS}

We are grateful to Roi Livni for helpful suggestions on the paper and to anonymous reviewers for helpful comments.

\section{REFERENCES}

[1] Jacob D. Abernethy, Young Hun Jung, Chansoo Lee, Audra McMillan, and Ambuj Tewari. 2019. Online Learning via the Differential Privacy Lens. In NeurIPS. 8892-8902.

[2] Naman Agarwal and Karan Singh. 2017. The Price of Differential Privacy for Online Learning. In ICML. 32-40.

[3] Noga Alon, Amos Beimel, Shay Moran, and Uri Stemmer. 2020. Closure Properties for Private Classification and Online Prediction. In COLT. 119-152.

[4] Noga Alon, Omri Ben-Eliezer, Yuval Dagan, Shay Moran, Moni Naor, and Eylon Yogev. 2021. Adversarial Laws of Large Numbers and Optimal Regret in Online Classification. In STOC.

[5] Noga Alon, Roi Livni, Maryanthe Malliaris, and Shay Moran. 2019. Private PAC Learning Implies Finite Littlestone Dimension. In STOC. 852-860.

[6] Peter L. Bartlett and Shahar Mendelson. 2003. Rademacher and Gaussian Com plexities: Risk Bounds and Structural Results. FMLR 3 (2003), 463-482.

[7] Amos Beimel, Hai Brenner, Shiva Prasad Kasiviswanathan, and Kobbi Nissim. 2014. Bounds on the sample complexity for private learning and private data release. Machine Learning 94 (2014), 401-437.

[8] Amos Beimel, Shay Moran, Kobbi Nissim, and Uri Stemmer. 2019. Private Center Points and Learning of Halfspaces. In COLT. 269-282.

[9] Amos Beimel, Kobbi Nissim, and Uri Stemmer. 2014. Private Learning and Sanitization: Pure vs. Approximate Differential Privacy. Theory of Computing 12 (07 2014).

[10] Amos Beimel, Kobbi Nissim, and Uri Stemmer. 2015. Learning Privately with Labeled and Unlabeled Examples. In SODA. 461-477.

[11] Amos Beimel, Kobbi Nissim, and Uri Stemmer. 2019. Characterizing the Sample Complexity of Pure Private Learners. FMLR 20, 146 (2019), 1-33.

[12] Shai Ben-David. 2015. 2 Notes on Classes with Vapnik-Chervonenkis Dimension 1. arXiv:1507.05307 (2015). arXiv:1507.05307

[13] Shai Ben-David, Dávid Pál, and Shai Shalev-Shwartz. 2009. Agnostic Online Learning. In COLT

[14] Siddharth Bhaskar. 2017. Thicket Density. arXiv:1702.03956 (2017)

[15] Aditya Bhaskara, Daniel Dadush, Ravishankar Krishnaswamy, and Kunal Talwar. 2012. Unconditional Differentially Private Mechanisms for Linear Queries. In STOC. $1269-1284$.

[16] Jaroslaw Blasiok, Mark Bun, Aleksandar Nikolov, and Thomas Steinke. 2019. Towards Instance-Optimal Private Query Release. In SODA. 2480-2497.

[17] Avrim Blum, Katrina Ligett, and Aaron Roth. 2008. A Learning Theory Approach to Non-Interactive Database Privacy. In STOC. 609-618.

[18] Olivier Bousquet, Roi Livni, and Shay Moran. 2020. Synthetic Data Generators: Sequential and Private. In NeurIPS.

[19] Mark Bun. 2020. A Computational Separation between Private Learning and Online Learning. In NeurIPS.

[20] Mark Bun, Cynthia Dwork, Guy N. Rothblum, and Thomas Steinke. 2018. Composable and Versatile Privacy via Truncated CDP. In STOC. 74-86.

[21] Mark Bun, Roi Livni, and Shay Moran. 2020. An equivalence between private classification and online prediction. In FOCS. 389-402.

[22] Mark Bun, Kobbi Nissim, and Uri Stemmer. 2016. Simultaneous Private Learning of Multiple Concepts. In ITCS. 369-380.

[23] Mark Bun, Kobbi Nissim, Uri Stemmer, and Salil P. Vadhan. 2015. Differentially Private Release and Learning of Threshold Functions. In FOCS. 634-649.

[24] Mark Bun, Jonathan Ullman, and Salil Vadhan. 2014. Fingerprinting Codes and the Price of Approximate Differential Privacy. In STOC. 1-10.
[25] Aloni Cohen and Kobi Nissim. 2020. Towards formalizing the GDPR's notion of singling out. PNAS 117(15) (2020).

[26] Richard M. Dudley. 1999. Uniform Central Limit Theorems. Cambridge University Press.

[27] Cynthia Dwork. 2006. Differential privacy. In ICALP. 1-12.

[28] Cynthia Dwork, Frank McSherry, Kobbi Nissim, and Adam Smith. 2006. Calibrating noise to sensitivity in private data analysis. In TCC. 265-284.

[29] Cynthia Dwork, Moni Naor, Omer Reingold, Guy N. Rothblum, and Salil Vadhan. 2009. On the Complexity of Differentially Private Data Release: Efficient Algorithms and Hardness Results. In STOC. 381-390.

[30] Cynthia Dwork and Aaron Roth. 2014. The Algorithmic Foundations of Differential Privacy. Now Publishers Inc.

[31] Cynthia Dwork, Guy N. Rothblum, and Salil Vadhan. 2010. Boosting and Differential Privacy. In FOCS. 51-60.

[32] Alexander Edmonds, Aleksandar Nikolov, and Jonathan Ullman. 2020. The Power of Factorization Mechanisms in Local and Central Differential Privacy. In STOC. 425-438.

[33] Vitaly Feldman and David Xiao. 2014. Sample Complexity Bounds on Differentially Private Learning via Communication Complexity. In COLT. 1-20.

[34] Badih Ghazi, Noah Golowich, Ravi Kumar, and Pasin Manurangsi. 2021. Sampleefficient proper private PAC learning. In $A L T$.

[35] Badih Ghazi, Ravi Kumar, and Pasin Manurangsi. 2020. Differentially Private Clustering: Tight Approximation Ratios. In NeurIPS.

[36] Alon Gonen, Elad Hazan, and Shay Moran. 2019. Private Learning Implies Online Learning: An Efficient Reduction. In NeurIPS. 8702-8712.

[37] Moritz Hardt, Katrina Ligett, and Frank McSherry. 2012. A Simple and Practical Algorithm for Differentially Private Data Release. In NIPS (Lake Tahoe, Nevada). 2339-2347.

[38] Moritz Hardt and Guy N. Rothblum. 2010. A Multiplicative Weights Mechanism for Privacy-Preserving Data Analysis. In FOCS. 61-70.

[39] Moritz Hardt and Kunal Talwar. 2010. On the Geometry of Differential Privacy. In STOC. $705-714$.

[40] Haim Kaplan, Katrina Ligett, Yishay Mansour, Moni Naor, and Uri Stemmer. 2020. Privately Learning Thresholds: Closing the Exponential Gap. In COLT. 2263-2285.

[41] Haim Kaplan, Yishay Mansour, Uri Stemmer, and Eliad Tsfadia. 2020. Private Learning of Halfspaces: Simplifying the Construction and Reducing the Sample Complexity. In NeurIPS

[42] Haim Kaplan, Micha Sharir, and Uri Stemmer. 2020. How to Find a Point in the Convex Hull Privately. In SoCG. 52:1-52:15.

[43] Shiva Prasad Kasiviswanathan, Homin K. Lee, Kobbi Nissim, Sofya Rashkodnikova, and Adam Smith. 2008. What can we Learn Privately?. In FOCS. 531-540.

[44] Nick Littlestone. 1987. Learning quickly when irrelevant attributes abound: A new linear-threshold algorithm. In FOCS. 68-77.

[45] Seth Neel, Aaron Roth, and Zhiwei Steven Wu. 2019. How to use heuristics for differential privacy. In FOCS. 72-93.

[46] A. Nikolov. 2015. An Improved Private Mechanism for Small Databases. In ICALP. $1010-1021$

[47] Aleksandar Nikolov, Kunal Talwar, and Li Zhang. 2012. The Geometry of Differential Privacy: the Sparse and Approximate Cases. STOC (2012), 351-360.

[48] Kobbi Nissim, Aaron Bembenek, Alexandra Wood, Mark Bun, Marco Gaboardi, Urs Gasser, David R. O’Brien, and Salil Vadhan. 2018. Bridging the Gap between Computer Science and Legal Approaches to Privacy. Harvard Journal of Law \& Technology 31 (2016 2018), 687-780. https://jolt.law.harvard.edu/assets/ articlePDFs/v31/02.-Article-Wood-7.21.pdf

[49] Article 29 Data Protection Working Party. 2014. Opinion 05/2014 on Anonymisation Techniques.

[50] Aaron Roth and Michael Kearns. 2019. The Ethical Algorithm: The Science of Socially Aware Algorithm Design. Oxford University Press.

[51] Aaron Roth and Tim Roughgarden. 2010. Interactive Privacy via the Median Mechanism. In STOC. 765-774.

[52] S. Shalev-Shwartz. 2012. Online Learning and Online Convex Optimization. Foundations and Trends in Machine Learning.

[53] Shai Shalev-Shwartz and Shai Ben-David. 2014. Understanding Machine Learning - From Theory to Algorithms. Cambridge University Press.

[54] Salil Vadhan. 2017. The complexity of differential privacy. In Tutorials on the Foundations of Cryptography. Springer, 347-450.

[55] Vladimir Vapnik. 1998. Statistical Learning Theory. Wiley-Interscience. 